\title{
SPATIAL RANDOM PERMUTATIONS WITH SMALL CYCLE WEIGHTS
}

\author{
VOLKER BETZ AND DANIEL UELTSCHI
}

\begin{abstract}
We consider the distribution of cycles in two models of random permutations, that are related to one another. In the first model, cycles receive a weight that depends on their length. The second model deals with permutations of points in the space and there is an additional weight that involves the length of permutation jumps. We prove the occurrence of infinite macroscopic cycles above a certain critical density.

Keywords: Random permutations, cycle weights, spatial permutations, infinite cycles.

2000 Math. Subj. Class.: 60K35, 82B20, 82B26, 82B41.
\end{abstract}

\section{INTRODUCTION}

Random permutations and their cycle structure have been studied for many decades, with a strongly increased activity in recent years. Apart from the rich mathematical structure, this interest is justified by a wide range of applications, from Gromov-Witten theory [10] to polynuclear growth [7] and to mathematical biology [5]. Detailed properties have been established in the case of uniform permutations for the moments of the distribution of the $n$-th shortest (or longest) cycle [13, for the longest increasing subsequence [1], or for the convergence to equilibrium [14. Physics and biology have suggested certain models with nonuniform permutations for which interesting results have been obtained [11, 6].

In the present work, we introduce a model for nonuniform permutations which is motivated by its connection to the theory of Bose-Einstein condensation [8, 15, 9, 2]. Mathematically, the distinguishing feature of our model, when compared to the works mentioned above, is that the measure on permutations possesses a spatial structure. More precisely, we consider pairs $(\boldsymbol{x}, \pi)$ with $\boldsymbol{x} \in \Lambda^{N}$ ( $\Lambda$ is a cubic box in $\mathbb{R}^{d}$ ) and $\pi \in \mathcal{S}_{N}$ (the group of permutations of $N$ elements). The weight of $(\boldsymbol{x}, \pi)$ is given by the "Gibbs factor" $\mathrm{e}^{-H(\boldsymbol{x}, \pi)}$ with Hamiltonian of the form

$$
H(\boldsymbol{x}, \pi)=\sum_{i=1}^{N} \xi\left(x_{i}-x_{\pi(i)}\right)+\sum_{\ell \geqslant 1} \alpha_{\ell} r_{\ell}(\pi) .
$$

We always assume that $\xi$ is a function $\mathbb{R}^{d} \rightarrow \mathbb{R} \cup\{\infty\}$, with $\int \mathrm{e}^{-\xi(x)} \mathrm{d}^{d} x=1$. The cycle parameters $\alpha_{1}, \alpha_{2}, \ldots$ are some fixed numbers, typically but not necessarily positive, and $r_{\ell}(\pi)$ is the number of cycles of length $\ell$ in the permutation $\pi$. The length of the cycle that contains the index $i$ is the smallest integer $\ell \geqslant 1$ such that $\pi^{\ell}(i)=i$ - this definition of the length involves the permutation but not the underlying spatial structure. Intuitively, the Gibbs factor restricts the permutations so each jump is local, i.e. the distances $\left|x_{i}-x_{\pi(i)}\right|$ remain finite even for large systems. The main question deals with the lengths of the cycles, in the limit of infinite volumes. 
When $\xi(x)=\gamma|x|^{2}+c$ and $\alpha_{\ell}=0$ for all $\ell$, we obtain the model of spatial random permutations that corresponds to the ideal Bose gas; in this case $\rho_{\mathrm{c}}$ is the well-known critical density for Bose-Einstein condensation for non-interacting particles. The occurrence of macroscopic cycles in the ideal Bose gas has been understood in [15, 16]. The present setting with general functions $\xi$ was considered in [2]. The latter article also introduces the cycle weight $\alpha_{2}$ as an approximation for the interactions between quantum particles, and the occurrence of macroscopic cycles was proved for large densities. The present article extends the results of [2] to more general cycle weights and to all densities larger than the critical density.

The main result of this article deals with the occurrence of infinite permutation cycles. Let $N_{k}(\pi)$ be the random variable that counts the number of points in cycles of length $k$ (we have $\sum_{k} N_{k}(\pi)=N$ for all $\pi$ ), and let $E_{\Lambda, N}\left(N_{k}\right)$ be its expectation. We consider the thermodynamic limit $|\Lambda|, N \rightarrow \infty$ with fixed density $\rho=N /|\Lambda|(|\Lambda|$ denotes the volume of $\Lambda$ ). Fatou's lemma implies that

$$
\sum_{k \geqslant 1} \lim _{|\Lambda| \rightarrow \infty} E_{\Lambda, \rho|\Lambda|}\left(\frac{N_{k}}{|\Lambda|}\right) \leqslant \rho .
$$

We prove in this article that the left side is strictly less than $\rho$ if and only if the density is larger than a critical density $\rho_{\mathrm{c}} \in(0, \infty]$. The precise formulation of this result can be found in Theorem 3.5. We need to restrict to certain functions $\xi$ (namely, $\mathrm{e}^{-\xi}$ has positive Fourier transform) and small cycle weights, in the sense that $\alpha_{\ell} \rightarrow 0$ as $\ell \rightarrow \infty$, faster than $1 / \log \ell$. Our results include an explicit formula for $\rho_{\mathrm{c}}$, cf. Eq. (3.8), and some characterization of the nature of infinite cycles - they are macroscopic and the distribution of a given macroscopic cycle is uniform in $\left[0, \rho-\rho_{\mathrm{c}}\right]$. Points that are not in macroscopic cycles are shown to be necessarily in finite cycles, and their density is given by $\max \left(\rho, \rho_{\mathrm{c}}\right)$. To our knowledge, the presence of a density of points in finite cycles is specific to spatial models, and does not occur in the other known models of random permutations.

The model considered here can be thought of as the "annealed" version of another "quenched" model where the space positions would be fixed. The latter model seems more attractive from a probability perspective, and it is actually discussed in [2]. There are two main advantages of the present annealed model: the availability of rigorous results about infinite cycles, and its closeness to the Feynman-Kac representation of the quantum Bose gas.

The structure of this article is as follows. In Section 2 we introduce an auxiliary model of non-spatial permutations with cycle weights, which turns out to be closely related to the spatial one. It corresponds to taking $\boldsymbol{x}=(0,0, \ldots)$. We only consider the case of small weights $\alpha_{\ell}$ so that the typical nonspatial permutations are like those with uniform distribution. We will study more general weights and other behaviors in a subsequent article 3 .

In Section 3 we introduce the model of spatial permutations with cycle weights. We discuss the existence of the infinite volume limits for thermodynamic potentials and the equivalence of ensembles. These notions belong to statistical mechanics rather than probability theory, but we need these results when we consider the more relevant question, as far as probability theory is concerned, of the occurrence of infinite cycles. Our main result is Theorem 3.5 in Section 3.2. We find in particular that the cycle weights modify the critical density; so they do have an effect on spatial permutations, unlike what was observed in Section 2. 
Section 4 is devoted to the proofs of the results about the thermodynamic potentials. We adapt the classical methods of Fisher and Ruelle [12] to our context. In Section 5 we relate our spatial model to an equivalent model in the Fourier representation, which allows to prove our main theorem. The nonspatial model with cycle weights also plays a rôle here. We use techniques introduced for the ideal Bose gas in [4] and [16].

\section{The SIMPle MOdel OF RANDOM PERMUtATIONS WITH CYCLE WEIGHT}

We start with the study of random permutations of $n$ elements with no spatial structure, but with cycle weights. The results of this section will be useful for the spatial model and this is our main motivation. But the present model has its own interest. We consider only small cycle weights here, but results for other regimes will be presented in a subsequent article [3].

2.1. Setting and properties. The sample space is $\mathcal{S}_{n}$ and the probability of a permutation $\pi \in \mathcal{S}_{n}$ is given by

$$
p_{n}(\pi)=\frac{1}{h_{n} n !} \exp \left\{-\sum_{\ell \geqslant 1} \alpha_{\ell} r_{\ell}(\pi)\right\}
$$

with normalization

$$
h_{n}=\frac{1}{n !} \sum_{\pi \in \mathcal{S}_{n}} \mathrm{e}^{-\sum_{\ell} \alpha_{\ell} r_{\ell}(\pi)} .
$$

Here, $\alpha_{1}, \alpha_{2}, \ldots$ are fixed numbers and $r_{\ell}(\pi)$ denotes the number of cycles of length $\ell$ in the permutation $\pi$. Notice the symmetry: since $\sum_{j} j r_{j}(\pi)=n$ for all $\pi$, the probability $p_{n}$ is invariant under the transformation

$$
\alpha_{j} \mapsto \alpha_{j}+c j,
$$

for any constant $c$; the normalization satisfies $h_{n} \mapsto \mathrm{e}^{-c n} h_{n}$.

Let $N_{a, b}(\pi)=\sum_{\ell=a}^{b} \ell r_{\ell}(\pi)$ denote the number of indices that belong to cycles of length between $a$ and $b$. Our main result deals with the asymptotic distribution of cycle lengths.

Theorem 2.1. If $\sum_{\ell \geqslant 1} \frac{1}{\ell}\left|1-\mathrm{e}^{-\alpha_{\ell}}\right|<\infty$, we have for any $0 \leqslant s \leqslant 1$

$$
\lim _{n \rightarrow \infty} \frac{1}{n} E_{n}\left(N_{1, s n}\right)=s .
$$

In essence, the hypothesis of the theorem requires that $\alpha_{\ell} \rightarrow 0$ a bit faster than $1 / \log \ell$. Theorem 2.1 implies that almost all indices belong to cycles whose length is a positive fraction of $n$. It can be shown that the number of cycles is of order $\log n$. The claim is easy to get in the case of uniform random permutations $\left(\alpha_{\ell} \equiv 0\right)$, but the extension to even small weights requires some efforts. The key to the proof of Theorem 2.1. which is given in the next subsection, is the following relation.

\section{Lemma 2.2.}

$$
E_{n}\left(N_{a, b}\right)=\sum_{j=a}^{b} \mathrm{e}^{-\alpha_{j}} \frac{h_{n-j}}{h_{n}} .
$$

For the proof of this lemma, we just remark that $E_{n}\left(N_{a, b}\right)=n p_{n}\left(\ell_{1} \in[a, b]\right)$, with $\ell_{1}=\ell_{1}(\pi)$ the length of the cycle that contains 1 . Summing over all possible values $j$ of $\ell_{1}$, and observing that there are $\frac{(n-1) !}{(n-j) !}$ possible cycles, we get the relation above. 
2.2. Properties of the normalization $h_{n}$. In view of Lemma 2.2 it is clear that we need to gather some information on $h_{n}$. We start with a few exact relations (Proposition 2.3 and we then obtain estimates (Proposition 2.4).

Proposition 2.3. The $h_{n}$ 's satisfy the following properties

(a) A recursion formula:

$$
h_{n}=\frac{1}{n} \sum_{\ell=1}^{n} \mathrm{e}^{-\alpha_{\ell}} h_{n-\ell}, \quad h_{0}=1 .
$$

(b) An explicit formula:

$$
h_{n}=\sum_{k=1}^{n} \frac{1}{k !} \sum_{\substack{\ell_{1}, \ldots, \ell_{k} \geqslant 1 \\ \ell_{1}+\ldots+\ell_{k}=n}} \prod_{i=1}^{k} \frac{\mathrm{e}^{-\alpha_{\ell_{i}}}}{\ell_{i}}, \quad n \geqslant 1 .
$$

(c) The increments satisfy

$$
h_{n}-h_{n-1}=\sum_{k=1}^{n} \frac{1}{k !} \sum_{\substack{\ell_{1}, \ldots, \ell_{k} \geqslant 1 \\ \ell_{1}+\ldots+\ell_{k}=n}} \prod_{i=1}^{k} \frac{\mathrm{e}^{-\alpha_{\ell_{i}}}-1}{\ell_{i}} .
$$

(d) Another formula for $h_{n}$ :

$$
h_{n}=\sum_{k=0}^{n} \frac{1}{k !} \sum_{\substack{\ell_{1}, \ldots, \ell_{k} \geqslant 1 \\ \ell_{1}+\ldots+\ell_{k} \leqslant n}} \prod_{i=1}^{k} \frac{\mathrm{e}^{-\alpha_{\ell_{i}}}-1}{\ell_{i}} .
$$

(e) If $\frac{\alpha_{\ell}}{\ell} \rightarrow 0$ and $\gamma>0$, we have

$$
\sum_{n \geqslant 0} \mathrm{e}^{-\gamma n} h_{n}=\exp \sum_{j \geqslant 1} \frac{\mathrm{e}^{-\gamma j-\alpha_{j}}}{j} .
$$

The formula (b) shows that $h_{n}$ is decreasing with respect to $\left(\alpha_{\ell}\right)$. The formula (d) shows that $h_{n}$ is increasing with respect to $n$ if $\alpha_{\ell} \leqslant 0$. Now we cannot resist but ask the reader to consider the following expression:

$$
\sum_{k=1}^{n} \frac{1}{k !} \sum_{\substack{\ell_{1}, \ldots, \ell_{k} \geqslant 1 \\ \ell_{1}+\cdots+\ell_{k}=n}} \frac{1}{\ell_{1} \ldots \ell_{k}}
$$

How does it behave for large $n$ ? The answer is surprisingly simple and is given by Proposition 2.3 (b).

Proof. The recursion formula is obtained from Lemma 2.2 by noting that $E_{n}\left(N_{1, n}\right)=n$. For the claim (b), it is useful to define $b_{j}=\mathrm{e}^{-\alpha_{j+1}}, j \geqslant 0$. The recursion formula can be written as

$$
(n+1) h_{n+1}=\sum_{j=0}^{n} b_{j} h_{n-j} .
$$

Thus the series $\left((n+1) h_{n+1}\right)$ is equal to the convolution of the series $\left(b_{n}\right)$ and $\left(h_{n}\right)$. We introduce the generating functions

$$
G_{h}(s)=\sum_{n \geqslant 0} h_{n} s^{n}, \quad G_{b}(s)=\sum_{n \geqslant 0} b_{n} s^{n} .
$$


The generating function for the series $\left((n+1) h_{n+1}\right)$ is $G_{h}^{\prime}(s)$. By the properties of convolutions, we have

$$
G_{h}^{\prime}(s)=G_{b}(s) G_{h}(s), \quad G_{h}(0)=1
$$

The solution is

$$
G_{h}(s)=\exp \int_{0}^{s} G_{b}(t) \mathrm{d} t=\exp \sum_{n \geqslant 1} \frac{b_{n-1}}{n} s^{n} .
$$

Expanding the exponential and rearranging the terms allows to find an expression for each coefficient $h_{n}$. This gives Proposition 2.3 (b).

The generating function for the increments, $G_{\delta h}$, satisfies

$$
G_{\delta h}(s) \doteqdot \sum_{n \geqslant 0}\left(h_{n}-h_{n-1}\right) s^{n}=(1-s) G_{h}(s)
$$

(with $h_{-1}=0$ ). Using (2.6), we get a differential equation for $G_{\delta h}$, namely

$$
G_{\delta h}^{\prime}(s)=G_{\delta h}(s)\left[G_{b}(s)-\frac{1}{1-s}\right]
$$

The expression in the bracket is equal to the generating function $G_{b-1}$ of the series $\left(b_{n}-1\right)$. Solving the differential equation, we get

$$
G_{\delta h}(s)=\exp \int_{0}^{s} G_{b-1}(t) \mathrm{d} t=\exp \sum_{n \geqslant 1} \frac{b_{n-1}-1}{n} s^{n} .
$$

Again expanding the exponential and matching the coefficients, we get Proposition 2.3 (c). The formula (d) follows from (c) since $h_{n}=\sum_{i=0}^{n}\left(h_{i}-h_{i-1}\right)$. Finally, (e) follows directly from $(2.7)$.

We now collect a few estimates for $h_{n}$.

\section{Proposition 2.4.}

(a) If $\sum_{\ell \geqslant 1} \frac{1}{\ell}\left|1-\mathrm{e}^{-\alpha_{\ell}}\right|<\infty$, we have $\sum_{n}\left|h_{n}-h_{n-1}\right|<\infty$, and

$$
h_{\infty} \doteqdot \lim _{n \rightarrow \infty} h_{n}=\exp \sum_{\ell \geqslant 1} \frac{\mathrm{e}^{-\alpha_{\ell}}-1}{\ell} .
$$

(b) $h_{n} \geqslant \frac{1}{n} h_{n-1} \mathrm{e}^{-\alpha_{1}}$; iterating, $h_{n} \geqslant \frac{1}{n !} \mathrm{e}^{-n \alpha_{1}}$.

(c) $h_{n} \geqslant \frac{1}{n} \mathrm{e}^{-\alpha_{n}}$.

(d) If $\left(\alpha_{\ell}\right)$ is subadditive (i.e. $\alpha_{\sum_{i} \ell_{i}} \leqslant \sum_{i} \alpha_{\ell_{i}}$ ), $h_{n} \leqslant \mathrm{e}^{-\alpha_{n}}$.

(e) If $\left(\alpha_{\ell}\right)$ is superadditive, $h_{n} \geqslant \mathrm{e}^{-\alpha_{n}}$.

Proof. The claim (a) is an immediate consequence of Proposition 2.3 (d) and the dominated convergence theorem: In the limit $n \rightarrow \infty$, the constraint $\ell_{1}+\ldots \ell_{k} \leqslant n$ vanishes and the corresponding expression factorizes. (b) and (c) follow from the recursion formula of Proposition 2.3 (a), keeping only the term $\ell=1$ for (b), and the term $\ell=n$ for (c). For (d) and (e), we write the formula of Proposition 2.3 (b) as

$$
h_{n}=\sum_{k=1}^{n} \frac{1}{k !} \sum_{\substack{\ell_{1}, \ldots, \ell_{k} \geqslant 1 \\ \ell_{1}+\cdots+\ell_{k}=n}} \frac{\mathrm{e}^{-\sum_{i=1}^{k} \alpha_{\ell_{i}}}}{\ell_{1} \ldots \ell_{k}} .
$$

We replace $\sum \alpha_{\ell_{i}}$ by $\alpha_{n}$, getting an upper bound for $h_{n}$ if $\left(\alpha_{\ell}\right)$ is subadditive, and a lower bound if $\left(\alpha_{\ell}\right)$ is superadditive. 
Proof of Theorem 2.1. We split the expression of Lemma 2.2 into

$$
\frac{1}{n} E\left(N_{1, s n}\right)=s+\sum_{j=1}^{s n} \frac{1}{n} \frac{h_{n-j}}{h_{n}}\left(\mathrm{e}^{-\alpha_{j}}-1\right)+\frac{1}{n} \sum_{j=1}^{s n}\left(\frac{h_{n-j}}{h_{n}}-1\right) .
$$

We suppose that $s n$ is an integer; it is easy to adapt the proof otherwise. Since $h_{n} \rightarrow h_{\infty}$, for any $\varepsilon>0$ there exists $n_{\varepsilon}$ such that if $n-j>n_{\varepsilon}$,

$$
1-\varepsilon<\frac{h_{n-j}}{h_{n}}<1+\varepsilon .
$$

The last term of (2.12) is then less than $\varepsilon$ if $s<1$ and $n$ large enough (the case $s=1$ is trivial). For any $\delta>0$, there exists $n_{\delta}$ such that $\sum_{j>n_{\delta}} \frac{1}{j}\left|1-\mathrm{e}^{-\alpha_{j}}\right|<\delta$. The sum over the first $n_{\delta}$ terms in the middle term of 2.12 is then less than $(1+\varepsilon) \frac{n_{\delta}}{n} \sum \frac{1}{j}\left|1-\mathrm{e}^{-\alpha_{j}}\right|$, and it vanishes in the limit $n \rightarrow \infty$; the sum over the remaining $n-n_{\delta}$ terms is less than $(1+\varepsilon) \delta$.

We conclude this section with a corollary which follows immediately from Proposition 2.4 and which will be very useful later.

Corollary 2.5. Let

$$
B=\sup _{m, n} \frac{h_{m}}{h_{n}} .
$$

Then if $\sum_{\ell \geqslant 1} \frac{1}{\ell}\left|1-\mathrm{e}^{-\alpha_{\ell}}\right|<\infty$, we have $0<B<\infty$.

\section{The SPATial MODEL OF RANDOM PERMUtATions}

We now introduce the spatial structure and consider the model described in the introduction. Let $\Lambda$ be an open bounded subset of $\mathbb{R}^{d}$, and let $N$ be the number of "particles" of the system. The state space of our model is

$$
\Omega_{\Lambda, N}=\Lambda^{N} \times \mathcal{S}_{N}
$$

with $\mathcal{S}_{N}$ the group of permutations of $N$ elements. $\Omega_{\Lambda, N}$ is equipped with the product of the Borel $\sigma$-algebra on $\Lambda^{N}$, and the discrete $\sigma$-algebra on $\mathcal{S}_{N}$. Let $(\boldsymbol{x}, \pi) \in \Omega_{\Lambda, N}$ with $\boldsymbol{x}=\left(x_{1}, \ldots, x_{N}\right)$; our Hamiltonian is given by (1.1).

3.1. The thermodynamic potentials. We consider the canonical ensemble where the particle density is fixed, and the grand-canonical ensemble where the chemical potential is fixed. The canonical partition function of this model is

$$
Y(\Lambda, N)=\frac{1}{N !} \int_{\Lambda^{N}} \mathrm{~d} \boldsymbol{x} \sum_{\pi \in \mathcal{S}_{N}} \mathrm{e}^{-H(\boldsymbol{x}, \pi)} .
$$

The division by $N$ ! guarantees that the partition function scales like the exponential of the volume. The definition makes sense for integer $N$; it is convenient to extend the partition function to noninteger $N$, e.g. by linear interpolation. The grand-canonical partition function is

$$
Z(\Lambda, \mu)=\sum_{N \geqslant 0} \mathrm{e}^{\mu N} Y(\Lambda, N)
$$


The parameter $\mu$ is called the chemical potential. Then we can define the two relevant thermodynamic potentials, the free energy and the pressure:

$$
\begin{aligned}
& q_{\Lambda}(\rho)=-\frac{1}{|\Lambda|} \log Y(\Lambda,|\Lambda| \rho), \\
& p_{\Lambda}(\mu)=\frac{1}{|\Lambda|} \log Z(\Lambda, \mu) .
\end{aligned}
$$

The parameter $\rho$ is the density.

We define the "dispersion relation" $\varepsilon(k)$ by

$$
\mathrm{e}^{-\varepsilon(k)}=\int_{\mathbb{R}^{d}} \mathrm{e}^{-2 \pi \mathrm{i} k x} \mathrm{e}^{-\xi(x)} \mathrm{d} x .
$$

For now $\varepsilon(k)$ can be complex; $\varepsilon(0)=0$ and $\operatorname{Re} \varepsilon(k) \geqslant a|k|^{2}$ for small $k$. The most relevant case is the Gaussian, $\mathrm{e}^{-\xi(x)}=(4 \pi \beta)^{-d / 2} \mathrm{e}^{-|x|^{2} / 4 \beta}$. This corresponds to the ideal Bose gas and $\varepsilon(k)=4 \pi^{2} \beta|k|^{2}$. We will suppose in the next subsection that $\varepsilon(k)$ is real.

Note also that the transformation $\alpha_{j} \mapsto \alpha_{j}+c j$ translates into

$$
\begin{aligned}
& q_{\Lambda}(\rho) \mapsto q_{\Lambda}(\rho)+c \rho ; \\
& p_{\Lambda}(\mu) \mapsto p_{\Lambda}(\mu-c) .
\end{aligned}
$$

Next we recall the notion of Fisher convergence [12]. A sequence $\left(\Lambda_{n}\right)$ of domains in $\mathbb{R}^{d}$ converges to $\mathbb{R}^{d}$ in the sense of Fisher if

- $\lim _{n \rightarrow \infty}\left|\Lambda_{n}\right|=\infty$.

- As $\varepsilon \rightarrow 0$,

$$
\sup _{n} \frac{\left|\partial_{\varepsilon \operatorname{diam} \Lambda_{n}} \Lambda_{n}\right|}{\left|\Lambda_{n}\right|} \longrightarrow 0
$$

where $\partial_{r} \Lambda=\left\{x \in \mathbb{R}^{d}: \operatorname{dist}(x, \partial \Lambda) \leqslant r\right\}$.

This notion is very general. If $\Lambda$ is bounded with piecewise smooth boundary, then the scaled domains $\Lambda_{n}=\{n x: x \in \Lambda\}$ form a Fisher sequence.

For the following three theorems, we always suppose that $\alpha_{\ell} / \ell$ converges as $\ell \rightarrow \infty$. Because of the symmetry (3.6) we can choose the limit, so we suppose that

$$
\lim _{\ell \rightarrow \infty} \frac{\alpha_{\ell}}{\ell}=0
$$

The first result is about the infinite volume pressure, that is given by an exact expression.

Theorem 3.1. For any $\mu \in \mathbb{R} \backslash\{0\}$, and any sequence $\Lambda_{n}$ that converges to $\mathbb{R}^{d}$ in the sense of Fisher, we have

$$
\lim _{n \rightarrow \infty} p_{\Lambda_{n}}(\mu)=p(\mu) \doteqdot \sum_{n \geqslant 1} \frac{\mathrm{e}^{\mu n-\alpha_{n}}}{n} \int_{\mathbb{R}^{d}} \mathrm{e}^{-n \varepsilon(k)} \mathrm{d} k .
$$

Notice that $p(\mu)$ is finite and analytic for $\mu<0$, and that $p(\mu)=\infty$ for $\mu>0$.

Next, we have the existence of the thermodynamic limit for the free energy.

Theorem 3.2. There exists a convex function $q(\rho)$ such that, for any sequence $\left(\Lambda_{n}\right)$ of domains converging to $\mathbb{R}^{d}$ (Fisher), and any sequence $\left(\rho_{n}\right)$ of numbers converging to $\rho \geqslant 0$, we have

$$
\lim _{n \rightarrow \infty} q_{\Lambda_{n}}\left(\rho_{n}\right)=q(\rho)
$$


Then $q$ is continuous. It is a standard exercise in analysis to show that the above property is equivalent to uniform convergence of $q_{n}$ to $q$ on compact intervals. Finally, pressure and free energy are related by Legendre transforms, a property known in statistical mechanics as "equivalence of ensembles".

Theorem 3.3. The infinite volume pressure and free energy are related as follows:

$$
\begin{aligned}
& q(\rho)=\sup _{\mu}[\rho \mu-p(\mu)], \\
& p(\mu)=\sup _{\rho}[\rho \mu-q(\rho)] .
\end{aligned}
$$

And $q(\rho)$ is analytic except at the critical density

$$
\rho_{\mathrm{c}}=\sum_{n \geqslant 1} \mathrm{e}^{-\alpha_{n}} \int_{\mathbb{R}^{d}} \mathrm{e}^{-n \varepsilon(k)} \mathrm{d} k .
$$

One can check that the critical density is real. It is always finite in dimensions $d \geqslant 3$; but it may be infinite in $d=1,2$, in which case $q$ is real analytic for all $\rho$ in $[0, \infty)$.
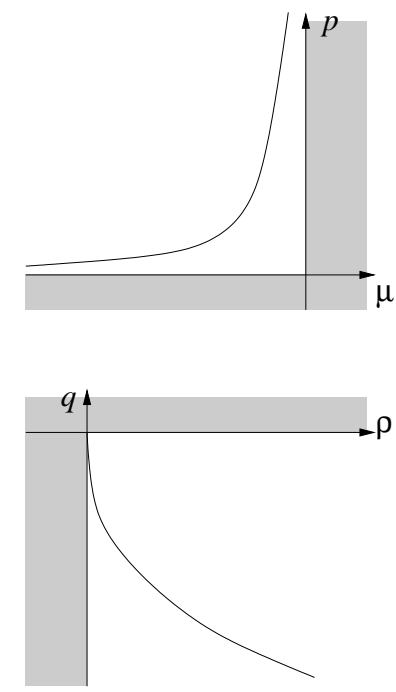

(a)
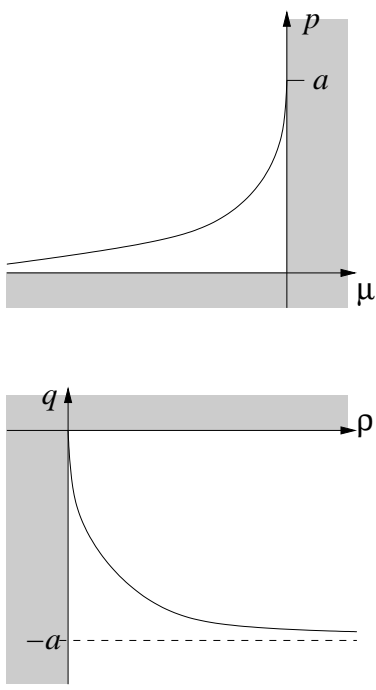

(b)
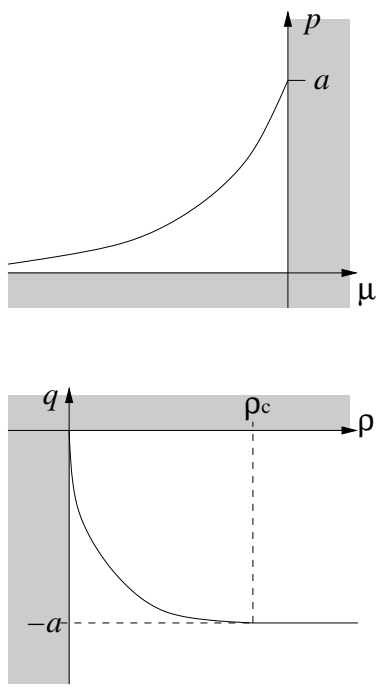

(c)

FIgURE 1. Qualitative graphs of the pressure $p(\mu)$ and its Legendre transform the free energy $q(\rho)$. The critical density $\rho_{\mathrm{c}}$ is infinite in (a) and (b), and it is finite in (c).

So far we have considered free boundary conditions. For the proofs of our results on the probability model below, we will need versions of the above theorems with periodized boundary conditions. To be precise, let us consider a sequence $\left(\Lambda_{n}\right)$ of $d$-dimensional boxes with side-length $L_{n}$, with $L_{n} \rightarrow \infty$. We define $H_{\Lambda}$ as in $(3.9)$ below, and let $q_{\Lambda}^{\text {per }}(\rho)$ and $p_{\Lambda}^{\text {per }}(\mu)$ be the corresponding free energy and pressure. As is usual in statistical mechanics, a change in boundary conditions brings a correction to thermodynamic potentials of the kind $q_{\Lambda}^{\text {per }}-q_{\Lambda} \approx \frac{|\partial \Lambda|}{|\Lambda|}$, with $|\partial \Lambda|$ a measure of the boundary of $\Lambda$; same for the pressure. The next theorem is less sharp but it is enough for our purpose. 
Theorem 3.4. For any $\rho \geqslant 0$ and any $\mu \in \mathbb{R} \backslash\{0\}$,

$$
\begin{aligned}
& \lim _{n \rightarrow \infty} q_{\Lambda_{n}}^{\text {per }}(\rho)=q(\rho) ; \\
& \lim _{n \rightarrow \infty} p_{\Lambda_{n}}^{\text {per }}(\mu)=p(\mu) .
\end{aligned}
$$

In both cases, convergence is uniform on compact intervals.

While Theorems 3.13 .3 are proved in Section 4 , Theorem 3.4 needs the notation of Section 5 and it is proved in the appendix.

3.2. The probability model. We will now study our model from a probabilistic point of view, proving the occurrence of infinite cycles above the critical density. As is often the case, we will need more stringent conditions than we did for studying the thermodynamic potentials.

We also need to slightly modify the Hamiltonian. Let $\Lambda$ be a $d$-dimensional cubic box with side length $L$ and volume $V=L^{d}$. Define $\xi_{\Lambda}$ through

$$
\mathrm{e}^{-\xi_{\Lambda}(x)}=\sum_{y \in \mathbb{Z}^{d}} \mathrm{e}^{-\xi(x-L y)}
$$

The important point is that $\mathrm{e}^{-\xi_{\Lambda}}$ has positive, $\Lambda$-independent Fourier transform $\mathrm{e}^{-\varepsilon(k)}$ already in finite volume; this helps us to relate our model with a probability model on Fourier modes. See Proposition 5.1 in Section 5. In particular, we note that $\int_{\Lambda} \mathrm{e}^{-\xi_{\Lambda}(x)} \mathrm{d}^{d} x=1$. If $\mathrm{e}^{-\xi}$ has compact support and if $L$ is larger than the diameter of the support, this "periodized" setting corresponds to usual periodic boundary conditions.

We now define

$$
H_{\Lambda}(\boldsymbol{x}, \pi)=\sum_{i=1}^{N} \xi_{\Lambda}\left(x_{i}-x_{\pi(i)}\right)+\sum_{\ell \geqslant 1} \alpha_{\ell} r_{\ell}(\pi) .
$$

and introduce a probability measure on $\Omega_{\Lambda, N}$ such that a random variable $\theta: \Omega_{\Lambda, N} \rightarrow \mathbb{R}$ has expectation

$$
E_{\Lambda, N}(\theta)=\frac{1}{Y(\Lambda, N) N !} \int_{\Lambda^{N}} \mathrm{~d} \boldsymbol{x} \sum_{\pi \in \mathcal{S}_{N}} \theta(\boldsymbol{x}, \pi) \mathrm{e}^{-H_{\Lambda}(\boldsymbol{x}, \pi)} .
$$

Note that $Y(\Lambda, N)$ is the partition function with periodized boundary conditions, a fact that we suppress from the notation.

Next, let $\ell_{i}(\pi)=1,2, \ldots$ denote the length of the permutation cycle of $\pi$ that contains the index $i$. It is convenient to consider the density of points that belong to cycles of certain lengths. Precisely, let

$$
\varrho_{a, b}(\pi)=\frac{1}{|\Lambda|} \#\left\{i=1,2, \cdots: a \leqslant \ell_{i}(\pi) \leqslant b\right\}=\frac{N_{a, b}(\pi)}{|\Lambda|} .
$$

Theorem 3.5. Assume that $\mathrm{e}^{-\xi}$ is continuous, that it has positive Fourier transform (i.e. $\varepsilon(k)$ is real), and that $\sum_{\ell \geqslant 1} \frac{\left|\alpha_{\ell}\right|}{\ell}<\infty$. We also suppose that $\rho_{\mathrm{c}}<\infty$. Let $\eta$ be any 
function such that $\eta(V) \rightarrow \infty$ and $\eta(V) / V \rightarrow 0$ as $V \rightarrow \infty$. Then for all $s \geqslant 0$,

$$
\begin{aligned}
& \lim _{V \rightarrow \infty} E_{\Lambda, \rho V}\left(\varrho_{1, \eta(V)}\right)=\left\{\begin{array}{ll}
\rho & \text { if } \rho \leqslant \rho_{\mathrm{c}} ; \\
\rho_{\mathrm{c}} & \text { if } \rho \geqslant \rho_{\mathrm{c}} ;
\end{array} \quad\right. \text { (microscopic cycles) } \\
& \lim _{V \rightarrow \infty} E_{\Lambda, \rho V}\left(\varrho_{\eta(V), V / \eta(V)}\right)=0 ; \quad \text { (mesoscopic cycles) } \\
& \lim _{V \rightarrow \infty} E_{\Lambda, \rho V}\left(\varrho_{\eta(V), s V}\right)= \begin{cases}0 & \text { if } \rho \leqslant \rho_{\mathrm{c}} ; \\
s & \text { if } 0 \leqslant s \leqslant \rho-\rho_{\mathrm{c}}, \quad \text { (macroscopic cycles) } \\
\rho-\rho_{\mathrm{c}} & \text { if } 0 \leqslant \rho-\rho_{\mathrm{c}} \leqslant s .\end{cases}
\end{aligned}
$$

Recall the expression (3.8) of the critical density. It is easily seen that

$$
\rho_{\mathrm{c}}=\infty \quad \Leftrightarrow \quad \int \frac{\mathrm{d} k}{\mathrm{e}^{\varepsilon(k)}-1}=\infty \quad \Leftrightarrow \quad \int_{|k|<1} \frac{\mathrm{d} k}{\varepsilon(k)}=\infty .
$$

The same theorem is stated in [2] but with $\alpha_{\ell} \equiv 0$; in this case it holds also when $\rho_{\mathrm{c}}=\infty$. The proof of Theorem 3.5, which can be found at the end of Section 5 , requires Proposition 5.4 which states that certain occupation numbers of Fourier modes are typical. But we can prove one of the claims of Proposition 5.4 only if $\rho_{\mathrm{c}}<\infty$. There is little doubt that all properties also hold true when the critical density is infinite.

\section{Thermodynamic Limits AND EQuivalence of Ensembles}

In this section we consider the thermodynamic limits of the pressure and of the free energy, and we prove Theorems 3.1 3.3. We adapt the methods devised in the 1960's by Fisher and Ruelle for classical particle systems. See [12] for references.

Proof of Theorem 3.1. The case $\alpha_{2} \neq 0, \alpha_{n}=0$ for any $n \neq 2$, was treated in [2]. The general case is similar. The key observation is that everything factorizes according to the permutation cycles.

The grand-canonical partition function reads

$$
Z(\Lambda, \mu)=\sum_{N \geqslant 0} \frac{\mathrm{e}^{\mu N}}{N !} \sum_{\pi \in \mathcal{S}_{N}} \prod_{n \geqslant 1}\left(\int_{\Lambda^{n}} \mathrm{~d} x_{1} \ldots \mathrm{d} x_{n} \mathrm{e}^{-\sum_{i=1}^{n} \xi\left(x_{i}-x_{i+1}\right)} \mathrm{e}^{-\alpha_{n}}\right)^{r_{n}(\pi)}
$$

where $r_{n}(\pi)$ is the number of cycles of length $n$ in the permutation $\pi$. We also supposed that $x_{n+1} \equiv x_{1}$. The number of permutations of $N$ elements with $r_{n}$ cycles of length $n$, $n \geqslant 1$, is equal to

$$
N ! / \prod_{n \geqslant 1} n^{r_{n}} r_{n} !
$$

Then

$$
\begin{aligned}
Z(\Lambda, \mu) & =\prod_{n \geqslant 1}\left\{\sum_{r \geqslant 0} \frac{1}{r !}\left(\frac{\mathrm{e}^{\mu n-\alpha_{n}}}{n} \int_{\Lambda^{n}} \mathrm{~d} x_{1} \ldots \mathrm{d} x_{n} \prod_{i=1}^{n} \mathrm{e}^{-\xi\left(x_{i}-x_{i+1}\right)}\right)^{r}\right\} \\
& =\exp \left\{\sum_{n \geqslant 1} \frac{\mathrm{e}^{\mu n-\alpha_{n}}}{n} \int_{\Lambda^{n}} \mathrm{~d} x_{1} \ldots \mathrm{d} x_{n} \prod_{i=1}^{n} \mathrm{e}^{-\xi\left(x_{i}-x_{i+1}\right)}\right\},
\end{aligned}
$$

and thus

$$
p_{\Lambda}(\mu)=\frac{1}{|\Lambda|} \sum_{n \geqslant 1} \frac{\mathrm{e}^{\mu n-\alpha_{n}}}{n} \int_{\Lambda^{n}} \mathrm{~d} x_{1} \ldots \mathrm{d} x_{n} \prod_{i=1}^{n} \mathrm{e}^{-\xi\left(x_{i}-x_{i+1}\right)}
$$


Now, with $x_{1}=0$ in the second term,

$$
\begin{aligned}
\frac{1}{|\Lambda|} \int_{\Lambda^{n}} \mathrm{~d} x_{1} \ldots \mathrm{d} x_{n} \prod_{i=1}^{n} \mathrm{e}^{-\xi\left(x_{i}-x_{i+1}\right)} & \leqslant \int_{\mathbb{R}^{d(n-1)}} \mathrm{d} x_{2} \ldots \mathrm{d} x_{n} \prod_{i=1}^{n} \mathrm{e}^{-\xi\left(x_{i}-x_{i+1}\right)} \\
& =\left(\mathrm{e}^{-\xi}\right)^{* n}(0) \\
& =\int_{\mathbb{R}^{d}} \mathrm{e}^{-n \varepsilon(k)} \mathrm{d} k .
\end{aligned}
$$

The inequality becomes an identity in the limit $\Lambda \nearrow \mathbb{R}^{d}$, and therefore we get Theorem 3.1 by dominated convergence if $\mu<0$. Since $\varepsilon(0)=0$ and $\varepsilon(k)>a|k|^{2}$, the integrals of $\mathrm{e}^{-n \varepsilon(k)}$ around $k=0$ decay as an inverse power of $n$, and the integral for large $k$ decays exponentially. Then if $\mu>0$, we have $p_{\Lambda}(\mu)=\infty$ for any $\Lambda$ large enough.

Let $F(\Lambda, N)$ denote the macroscopic free energy,

$$
F(\Lambda, N)=-\log Y(\Lambda, N)
$$

Lemma 4.1. Let $\Lambda_{1}, \Lambda_{2}$ be disjoint open bounded subsets of $\mathbb{R}^{d}$. Then for any integers $N_{1}, N_{2}$,

$$
F\left(\Lambda_{1} \cup \Lambda_{2}, N_{1}+N_{2}\right) \leqslant F\left(\Lambda_{1}, N_{1}\right)+F\left(\Lambda_{2}, N_{2}\right) .
$$

Among the many useful consequences of this subadditive property, we get the upper bound:

$$
F(\Lambda, N) \leqslant \sum_{i=1}^{N} F\left(\Lambda_{i}, 1\right)=N\left(\xi(0)+\alpha_{1}+\log \rho\right)
$$

where the $\Lambda_{i}$ 's partition $\Lambda$ in subdomains, each of volume $|\Lambda| / N=1 / \rho$.

Proof. We show that $Y\left(\Lambda_{1} \cup \Lambda_{2}, N_{1}+N_{2}\right) \geqslant Y\left(\Lambda_{1}, N_{1}\right) Y\left(\Lambda_{2}, N_{2}\right)$. From the definition,

$$
Y\left(\Lambda_{1} \cup \Lambda_{2}, N_{1}+N_{2}\right)=\frac{1}{\left(N_{1}+N_{2}\right) !} \int_{\left(\Lambda_{1} \cup \Lambda_{2}\right)^{N_{1}}} \mathrm{~d} \boldsymbol{x} \int_{\left(\Lambda_{1} \cup \Lambda_{2}\right)^{N_{2}}} \mathrm{~d} \boldsymbol{y} \sum_{\pi \in \mathcal{S}_{N_{1}+N_{2}}} \mathrm{e}^{-H(\boldsymbol{x} \times \boldsymbol{y}, \pi)} .
$$

We get an upper bound by restricting the integrals so that exactly $N_{1}$ points fall in $\Lambda_{1}$ and $N_{2}$ points in $\Lambda_{2}$. Rearranging the integrals, we obtain

$$
Y\left(\Lambda_{1} \cup \Lambda_{2}, N_{1}+N_{2}\right) \geqslant \frac{1}{N_{1} ! N_{2} !} \int_{\Lambda_{1}^{N_{1}}} \mathrm{~d} \boldsymbol{x} \int_{\Lambda_{2}^{N_{2}}} \mathrm{~d} \boldsymbol{y} \sum_{\pi \in \mathcal{S}_{N_{1}+N_{2}}} \mathrm{e}^{-H(\boldsymbol{x} \times \boldsymbol{y}, \pi)} .
$$

We restrict the sum to permutations of the kind $\pi=\pi_{1} \times \pi_{2}$, with $\pi_{1}$ a permutation of the first $N_{1}$ elements and $\pi_{2}$ a permutation of the last $N_{2}$ elements. Then

$$
Y\left(\Lambda_{1} \cup \Lambda_{2}, N_{1}+N_{2}\right) \geqslant \frac{1}{N_{1} ! N_{2} !} \int_{\Lambda_{1}^{N_{1}}} \mathrm{~d} \boldsymbol{x} \int_{\Lambda_{2}^{N_{2}}} \mathrm{~d} \boldsymbol{y} \sum_{\substack{\pi_{1} \in \mathcal{S}_{N_{1}} \\ \pi_{2} \in \mathcal{S}_{N_{2}}}} \mathrm{e}^{-H\left(\boldsymbol{x} \times \boldsymbol{y}, \pi_{1} \times \pi_{2}\right)} .
$$

Finally, we observe that

$$
H\left(\boldsymbol{x} \times \boldsymbol{y}, \pi_{1} \times \pi_{2}\right)=H\left(\boldsymbol{x}, \pi_{1}\right)+H\left(\boldsymbol{y}, \pi_{2}\right) .
$$

Integrals in 4.9 factorize, yielding the product of partition functions.

Next we identify the free energy by considering a special sequence of increasing domains. 
Lemma 4.2. Let $C_{n}$ be the cube of size $2^{n}$ centered at the origin, and define

$$
q(\rho)=\lim _{n \rightarrow \infty} 2^{-d n} F\left(C_{n},\left\lfloor 2^{n} \rho\right\rfloor\right) .
$$

Then

(a) the above limit exists indeed.

(b) $q(\rho)$ is convex.

Proof. The existence of the limit follows from a standard subadditive argument, where we show that

$$
\limsup _{n \rightarrow \infty} 2^{-d n} F\left(C_{n},\left\lfloor 2^{n} \rho\right\rfloor\right) \leqslant \liminf _{n \rightarrow \infty} 2^{-d n} F\left(C_{n},\left\lfloor 2^{n} \rho\right\rfloor\right) .
$$

Fix $k$; we consider $n>2 k$. The cube $C_{n}$ can be partitioned into $2^{d(n-k)}$ cubes $C_{k}$. We put $\left\lfloor 2^{d k} \rho\right\rfloor$ points in the first $2^{d(n-k)}-2^{d(n-2 k)}$ cubes, and the remaining $N_{n k}$ points in the remaining $2^{d(n-2 k)}$ cubes. By subadditivity, and the upper bound 4.6 ), we get

$$
F\left(C_{n},\left\lfloor 2^{d n} \rho\right\rfloor\right) \leqslant\left(2^{d(n-k)}-2^{d(n-2 k)}\right) F\left(C_{k},\left\lfloor 2^{d k} \rho\right\rfloor\right)+N_{n k}\left(\xi(0)+\alpha_{1}+\log \frac{N_{n k}}{2^{d(n-k)}}\right) .
$$

The number $N_{n k}$ is not too big:

$$
N_{n k}=\left\lfloor 2^{d n} \rho\right\rfloor-\left(2^{d(n-k)}-2^{d(n-2 k)}\right)\left\lfloor 2^{d k} \rho\right\rfloor \leqslant 2^{d(n-k)}(1+\rho) .
$$

Then

$$
\limsup _{n \rightarrow \infty} 2^{-d n} F\left(C_{n},\left\lfloor 2^{n} \rho\right\rfloor\right) \leqslant 2^{-d k}\left(1-2^{-d k}\right) F\left(C_{k},\left\lfloor 2^{d k} \rho\right\rfloor\right)+2^{-d k}(1+\rho)\left(\xi(0)+\alpha_{1}+\log (1+\rho)\right) .
$$

Taking the liminf $k \rightarrow \infty$ in the right side, we obtain (4.11).

We now prove that $q(\rho)$ satisfies a certain form a continuity, see 4.20 below. Let $\varepsilon>0$; we study

$$
q(\rho+\varepsilon)=\lim _{n} 2^{-d n} F\left(C_{n},\left\lfloor 2^{d n}(\rho+\varepsilon)\right\rfloor\right) .
$$

Fix $k$. There are $2^{d(n-k)}$ cubes $C_{k}$ in $C_{n}$. Put $\left\lfloor 2^{d k} \rho\right\rfloor$ points in the first $\left\lfloor 2^{d(n-k)}(1-\varepsilon)\right\rfloor$ cubes, and the remaining $N_{n k}$ points in the remaining domain $D_{n k}$. By subadditivity and the upper bound 4.6,

$$
F\left(C_{n},\left\lfloor 2^{d n}(\rho+\varepsilon)\right\rfloor\right) \leqslant\left\lfloor 2^{d(n-k)}(1-\varepsilon)\right\rfloor F\left(C_{k},\left\lfloor 2^{d k} \rho\right\rfloor\right)+N_{n k}\left(\xi(0)+\alpha_{1}+\log \frac{N_{n k}}{\left|D_{n k}\right|}\right) .
$$

We can estimate the last term:

$$
\begin{aligned}
& \left.N_{n k}=\left\lfloor 2^{d n}(\rho+\varepsilon)\right\rfloor-\left\lfloor 2^{d(n-k)}(1-\varepsilon)\right\rfloor 2^{d k} \rho\right\rfloor \leqslant 2^{d n+1} \varepsilon+2^{d(n-k)}+2^{d k} \rho, \\
& \left|D_{n k}\right|=2^{d n}-2^{d k}\left\lfloor 2^{d(n-k)}(1-\varepsilon)\right\rfloor \geqslant 2^{d n} \varepsilon .
\end{aligned}
$$

Then

$N_{n k}\left(\xi(0)+\alpha_{1}+\log \frac{N_{n k}}{\left|D_{n k}\right|}\right) \leqslant 2^{d n}\left(2 \varepsilon+2^{-k}+2^{-d(n-k)} \rho\right)\left(\xi(0)+\alpha_{1}+\log \left(2+\frac{2^{-d k}}{\varepsilon}+\frac{2^{-d(n-k)}}{\varepsilon}\right)\right)$.

We substitute these bounds into 4.16, divide by $2^{d n}$, and let $n \rightarrow \infty$. We get

$$
q(\rho+\varepsilon) \leqslant 2^{-d k}(1-\varepsilon) F\left(C_{k},\left\lfloor 2^{d k} \rho\right\rfloor\right)+\left(2 \varepsilon+2^{-k}\right)\left(\xi(0)+\alpha_{1}+\log \left(2+\frac{2^{-d k}}{\varepsilon}\right)\right) .
$$

This is true for any $k$. As $k \rightarrow \infty$,

$$
q(\rho+\varepsilon) \leqslant(1-\varepsilon) q(\rho)+2 \varepsilon\left(\xi(0)+\alpha_{1}+\log 2\right) .
$$


Finally, we prove a weak version of convexity. Combined with 4.20 , it implies that $q(\rho)$ is convex indeed. For any $\rho_{1}$ and $\rho_{2}$ such that $2^{d n-1} \rho_{i}$ is integer for $n$ large enough,

$$
\begin{aligned}
q\left(\frac{1}{2} \rho_{1}+\frac{1}{2} \rho_{2}\right) & =\lim _{n} 2^{-d n} F\left(C_{n}, 2^{d n-1} \rho_{1}+2^{d n-1} \rho_{2}\right) \\
& \leqslant \lim _{n} 2^{-d n}\left[2^{d-1} F\left(C_{n-1}, 2^{d(n-1)} \rho_{1}\right)+2^{d-1} F\left(C_{n-1}, 2^{d(n-1)} \rho_{2}\right)\right] \\
& =\frac{1}{2} q\left(\rho_{1}\right)+\frac{1}{2} q\left(\rho_{2}\right) .
\end{aligned}
$$

Indeed, we put a density $\rho_{1}$ of points in half the cubes $C_{n-1}$, and a density $\rho_{2}$ in the other half, and we used subadditivity.

We can now prove Theorem 3.2 about the convergence of the thermodynamic limit for the free energy.

Proof of Theorem 3.2. First, we show that

$$
\limsup _{n \rightarrow \infty} \frac{1}{\left|\Lambda_{n}\right|} F\left(\Lambda_{n},\left|\Lambda_{n}\right| \rho_{n}\right) \leqslant q(\rho)
$$

Choose a cube $C$, and a number $\rho^{\prime}>\rho$. Given $n$, let $M_{n}$ be the largest integer such that $M_{n}|C| \rho^{\prime} \leqslant\left|\Lambda_{n}\right| \rho_{n}$. We pave $\mathbb{R}^{d}$ with translates of the cube $C$. The volume of cubes inside $\Lambda_{n}$ is at least $\left|\Lambda_{n}\right|-\left|\partial_{\text {diam } C} \Lambda_{n}\right|$. Thus for $n$ large enough, the number of cubes inside $\Lambda_{n}$ is at least

$$
\frac{\left|\Lambda_{n}\right|-\left|\partial_{\operatorname{diam} C} \Lambda_{n}\right|}{|C|} \geqslant \frac{\left|\Lambda_{n}\right|}{|C|} \frac{\rho_{n}}{\rho^{\prime}} \geqslant M_{n} .
$$

By subadditivity of the free energy,

$$
F\left(\Lambda_{n},\left|\Lambda_{n}\right| \rho_{n}\right) \leqslant M_{n} F(C,\lfloor|C| \rho\rfloor)+F(D, N)
$$

with

$$
F(D, N) \leqslant N\left(\alpha_{1}+\log \frac{N}{|D|}\right) .
$$

Now

$$
\begin{aligned}
N & =\left|\Lambda_{n}\right| \rho_{n}-M_{n}\lfloor|C| \rho\rfloor \leqslant\left|\Lambda_{n}\right| \rho_{n}\left(1-\frac{\rho}{\rho^{\prime}}+\frac{1}{|C| \rho^{\prime}}\right)+|C| \rho, \\
|D| & =\left|\Lambda_{n}\right|-M_{n}|C| \geqslant\left|\Lambda_{n}\right|\left(1-\frac{\rho_{n}}{\rho^{\prime}}\right) .
\end{aligned}
$$

Then

$$
\begin{aligned}
\frac{1}{\left|\Lambda_{n}\right|} F\left(\Lambda_{n},\left|\Lambda_{n}\right| \rho_{n}\right) \leqslant & \frac{M_{n}|C|}{\left|\Lambda_{n}\right|} \frac{1}{|C|} F(C,\lfloor|C| \rho\rfloor) \\
& +\left(\rho_{n}\left(1-\frac{\rho}{\rho^{\prime}}-\frac{1}{|C| \rho^{\prime}}\right)+\frac{|C| \rho}{\left|\Lambda_{n}\right|}\right)\left(\alpha_{1}+\log \frac{\rho_{n}\left(1-\frac{\rho}{\rho^{\prime}}+\frac{1}{|C| \rho^{\prime}}\right)+\frac{|C|}{\left|\Lambda_{n}\right|}}{1-\frac{\rho}{\rho^{\prime}}}\right) .
\end{aligned}
$$

Letting $n \rightarrow \infty$, the expression simplifies a bit:

$$
\limsup _{n \rightarrow \infty} \frac{1}{\left|\Lambda_{n}\right|} F\left(\Lambda_{n},\left|\Lambda_{n}\right| \rho_{n}\right) \leqslant \frac{\rho}{\rho^{\prime}} \frac{1}{|C|} F(C,\lfloor|C| \rho\rfloor)+\rho\left(1-\frac{\rho}{\rho^{\prime}}-\frac{1}{|C| \rho^{\prime}}\right)\left(\alpha_{1}+\log \frac{\rho\left(1-\frac{\rho}{\rho^{\prime}}+\frac{1}{|C| \rho^{\prime}}\right)}{1-\frac{\rho}{\rho^{\prime}}}\right) .
$$

We can consider the cubes $C_{k}$ of Lemma 4.2 and take the limit $k \rightarrow \infty$. Then we let $\rho^{\prime} \rightarrow \rho$, and we get 4.22 .

We complete the proof by showing the complementary lower bound, namely

$$
\liminf _{n \rightarrow \infty} \frac{1}{\left|\Lambda_{n}\right|} F\left(\Lambda_{n},\left|\Lambda_{n}\right| \rho_{n}\right) \geqslant q(\rho) .
$$


A consequence of the limit in the sense of Fisher is that, given $\Lambda_{n} \nearrow \mathbb{R}^{d}$, there exists $\eta>0$ such that each $\Lambda_{n}$ contains a translate of a cube of size $\eta \operatorname{diam} \Lambda_{n}$. Given $n$, let $k$ be such that $2^{k} \geqslant \operatorname{diam} \Lambda_{n}>2^{k-1}$. Then $\Lambda_{n}$ is contained in a translate of $C_{k}$, and

$$
1 \geqslant \frac{\left|\Lambda_{n}\right|}{\left|C_{k}\right|} \geqslant 2^{-d k}\left(\eta \operatorname{diam} \Lambda_{n}\right)^{d} \geqslant 2^{-d k}\left(\eta 2^{k-1}\right)^{d}=\left(\frac{1}{2} \eta\right)^{d} .
$$

By subadditivity, we have

$$
F\left(C_{k},\left\lfloor 2^{d k} \rho\right\rfloor\right) \leqslant F\left(\Lambda_{n},\left|\Lambda_{n}\right| \rho_{n}\right)+F\left(C_{k} \backslash \Lambda_{n},\left\lfloor 2^{d k} \rho\right\rfloor-\left|\Lambda_{n}\right| \rho_{n}\right) .
$$

As $n \rightarrow \infty$, we also have $k \rightarrow \infty$. Then, taking the lim inf of the expression above, we get

$$
\begin{aligned}
\liminf _{n \rightarrow \infty} \frac{1}{\left|\Lambda_{n}\right|} F\left(\Lambda_{n},\left|\Lambda_{n}\right| \rho_{n}\right) \geqslant \liminf _{n \rightarrow \infty}\left[\frac{2^{d k}}{\left|\Lambda_{n}\right|} 2^{-d k} F\left(C_{k},\left\lfloor 2^{d k} \rho\right\rfloor\right)\right. \\
\left.\quad-\frac{2^{d k}-\left|\Lambda_{n}\right|}{\left|\Lambda_{n}\right|} \frac{1}{2^{d k}-\left|\Lambda_{n}\right|} F\left(C_{k} \backslash \Lambda_{n},\left\lfloor 2^{d k} \rho\right\rfloor-\left|\Lambda_{n}\right| \rho_{n}\right)\right] .
\end{aligned}
$$

Notice that $\left\lfloor 2^{d k} \rho\right\rfloor-\left|\Lambda_{n}\right| \rho_{n} \equiv\left|C_{k} \backslash \Lambda_{n}\right| \rho_{n}^{\prime}$ with

$$
\rho_{n}^{\prime}=\frac{\left\lfloor 2^{d k} \rho\right\rfloor-\left|\Lambda_{n}\right| \rho_{n}}{\left|C_{k} \backslash \Lambda_{n}\right|} \longrightarrow \rho
$$

as $n \rightarrow \infty$. This uses (4.30). Using the result for the limsup, Eq. 4.22), we find that the right side of 4.32 is larger than $q(\rho)$.

There remains to prove Theorem 3.3. It is actually enough to show that $p$ is the Legendre transform of $q$ - since $q$ is convex, it is necessarily the Legendre transform of $p$.

Proof of Theorem 3.3. From 3.3 and 3.4, we have

$$
p_{\Lambda}(\mu)=\frac{1}{|\Lambda|} \log \sum_{\rho \in \mathbb{N} /|\Lambda|} \exp \left\{|\Lambda|\left[\rho \mu-q_{\Lambda}(\rho)\right]\right\} .
$$

By restricting the sum over a single $\rho$, we get

$$
p_{\Lambda}(\mu) \geqslant \rho \mu-q_{\Lambda}(\rho) .
$$

This holds for any $\Lambda$ and any $\rho$ such that $|\Lambda| \rho$ is integer. It follows that for any $\rho>0$, we can use a suitable Fisher sequence (such as cubic boxes of size $L=\rho^{-1 / d} k, k \in \mathbb{N}$ ) so as to obtain

$$
p(\mu) \geqslant \rho \mu-q(\rho) .
$$

This inequality also holds when taking the supremum over $\rho$ in the right side.

The upper bound requires a bit more work. By (4.35), we have

$$
\rho \mu-q_{\Lambda}(\rho)=\frac{1}{2} \rho \mu+\frac{1}{2} \rho \mu-q_{\Lambda}(\rho) \leqslant \frac{1}{2} \rho \mu+p_{\Lambda}\left(\frac{\mu}{2}\right) .
$$

Let $A$ be a number that is independent of $\Lambda$, to be determined later. Then

$$
p_{\Lambda}(\mu) \leqslant \frac{1}{|\Lambda|} \log \left\{\sum_{\substack{\rho \in \mathbb{N} /|\Lambda| \\ \rho \leqslant A}} \mathrm{e}^{|\Lambda|\left[\rho \mu-q_{\Lambda}(\rho)\right]}+\sum_{\substack{\rho \in \mathbb{N} /|\Lambda| \\ \rho>A}} \mathrm{e}^{|\Lambda|\left[\frac{1}{2} \rho \mu+p_{\Lambda}\left(\frac{\mu}{2}\right)\right]}\right\} .
$$

The latter sum is equal to $\mathrm{e}^{|\Lambda| p_{\Lambda}\left(\frac{\mu}{2}\right)} \mathrm{e}^{\frac{1}{2} \mu\lceil A|\Lambda|\rceil}\left(1-\mathrm{e}^{\frac{1}{2} \mu}\right)^{-1}$.

It follows from Theorem 3.2 that $q_{\Lambda}(\rho)$ converges to $q(\rho)$ uniformly on compact intervals. Thus for any $\varepsilon>0$, there exists $\Lambda$ large enough so that

$$
\rho \mu-q_{\Lambda}(\rho) \leqslant \rho^{*} \mu-q\left(\rho^{*}\right)+\varepsilon,
$$


for any $0<\rho<A$. Then

$$
p_{\Lambda}(\mu) \leqslant \rho^{*} \mu-q\left(\rho^{*}\right)+\varepsilon+\frac{1}{|\Lambda|} \log \left\{A|\Lambda|+\left(1-\mathrm{e}^{\frac{1}{2} \mu}\right)^{-1} \mathrm{e}^{|\Lambda|\left[p_{\Lambda}\left(\frac{\mu}{2}\right)-\rho^{*} \mu+q\left(\rho^{*}\right)-\varepsilon+\frac{1}{2} A \mu\right]}\right\} .
$$

We can choose $A$ large enough such that the exponent is negative. Letting $\Lambda \nearrow \mathbb{R}^{d}$, we see that $p(\mu) \leqslant \sup _{\rho}[\rho \mu-q(\rho)]$ indeed.

\section{The Fourier MOdel OF RANDOM PERMUtATiONS}

We consider now the setting of Section 3.2 . Thus $\Lambda$ is a cube of side length $L$ and volume $V=L^{d}$. Let $\Lambda^{*}=\frac{1}{L} \mathbb{Z}^{d}$ denote the dual space. The goal of this section is to describe a probability model on $\Omega_{\Lambda, N}^{*}=\left(\Lambda^{*}\right)^{N} \times \mathcal{S}_{N}$, whose marginal distribution on permutations coincide with the model of spatial permutations. The method is inspired by [16], who studied the nature of cycles in the ideal Bose gas. The beginning of this section is almost identical to [2, the difference being that $\alpha_{\ell} \neq 0$ here.

For a bounded domain $\Lambda$ the new probability space is discrete. We give ourselves a positive continuous function $\varepsilon(k), k \in \mathbb{R}^{d}$. We suppose that $\varepsilon(0)=0$, and that $\varepsilon(k) \geqslant a|k|^{2}$ for small $k$. The probability of $(\boldsymbol{k}, \pi) \in \Omega_{\Lambda, N}^{*}$ is defined by

$$
p_{\Lambda, N}(\boldsymbol{k}, \pi)= \begin{cases}\frac{1}{\widehat{Y}(\Lambda, N) N !} \mathrm{e}^{-\widehat{H}(\boldsymbol{k}, \pi)} & \text { if } k_{i}=k_{\pi(i)} \text { for all } i, \\ 0 & \text { otherwise. }\end{cases}
$$

Here, the Hamiltonian $\widehat{H}$ is given by

$$
\widehat{H}(\boldsymbol{k}, \pi)=\sum_{i=1}^{N} \varepsilon\left(k_{i}\right)+\sum_{\ell \geqslant 1} \alpha_{\ell} r_{\ell}(\pi) .
$$

This model offers an alternate representation to the model of spatial permutations, as far as the permutations are concerned, thanks to the following relation. Let $\delta_{i, j}$ denote Kronecker's delta symbol.

Proposition 5.1. Suppose that $\widehat{\mathrm{e}^{-\xi}}=\mathrm{e}^{-\varepsilon}$, and define $H_{\Lambda}$ through $(3.9)$. Then, for any permutation $\pi$,

$$
\int_{\Lambda^{N}} \mathrm{e}^{-H_{\Lambda}(\boldsymbol{x}, \pi)} \mathrm{d} \boldsymbol{x}=\sum_{\boldsymbol{k} \in\left(\Lambda^{*}\right)^{N}} \mathrm{e}^{-\widehat{H}(\boldsymbol{k}, \pi)} \prod_{i=1}^{N} \delta_{k_{i}, k_{\pi(i)}} .
$$

In particular, it follows that $\widehat{Y}(\Lambda, N)=Y(\Lambda, N)$, and $E_{\Lambda, N}\left(\Lambda^{N} \times\{\pi\}\right)=p_{\Lambda, N}\left(\Omega_{\Lambda, N}^{*}, \pi\right)$ for all permutations $\pi$.

Proof. We use the following identity, which follows almost directly from well-known relations between Fourier transform and convolution:

$$
\int_{\Lambda^{N}} \mathrm{~d} \boldsymbol{x} \prod_{i=1}^{N} \mathrm{e}^{-\xi_{\Lambda}\left(x_{i}-x_{\pi(i)}\right)}=\sum_{\boldsymbol{k} \in\left(\Lambda^{*}\right)^{N}} \prod_{i=1}^{N}\left[\delta_{k_{i}, k_{\pi(i)}} \mathrm{e}^{-\varepsilon\left(k_{i}\right)}\right] .
$$

See [2], Corollary 5.3, for more details. Notice that the case $N=1$ reduces to Poisson summation formula, and it holds true because $\mathrm{e}^{-\xi}$ is continuous. Multiplying both sides by $\mathrm{e}^{-\sum \alpha_{\ell} r_{\ell}(\pi)}$, one gets the result. 
Next, we introduce occupation numbers. Let $\mathcal{N}_{\Lambda}$ be the set of sequences $\boldsymbol{n}=\left(n_{k}\right)$ of integers indexed by $k \in \Lambda^{*}$, and let $\mathcal{N}_{\Lambda, N}$ the set of occupation numbers with total number $N$ :

$$
\mathcal{N}_{\Lambda, N}=\left\{\boldsymbol{n} \in \mathcal{N}_{\Lambda}: \sum_{k \in \Lambda^{*}} n_{k}=N\right\}
$$

To each $\boldsymbol{k} \in\left(\Lambda^{*}\right)^{N}$ corresponds an element $\boldsymbol{n} \in \mathcal{N}_{\Lambda, N}$, with $n_{k}$ counting the number of indices $i$ such that $k_{i}=k$. Thus we can view $\boldsymbol{n}$ as a subset of $\left(\Lambda^{*}\right)^{N}$. The probability (5.1) yields a probability on occupation numbers. Indeed, summing over permutations and over compatible vectors $\boldsymbol{k}$, we have

$$
p_{\Lambda, N}(\boldsymbol{n})=\frac{1}{Y(\Lambda, N)} \prod_{k \in \Lambda^{*}} \mathrm{e}^{-n_{k} \varepsilon(k)} h_{n_{k}}
$$

with $h_{n}$ defined in 2.2 .

We will obtain some properties on the probability $p_{\Lambda, N}(\boldsymbol{n})$ below. But we first relate this probability with the lengths of permutation cycles.

\section{Proposition 5.2.}

$$
E_{\Lambda, N}\left(\varrho_{a, b}\right)=\frac{1}{V} \sum_{\boldsymbol{n} \in \mathcal{N}_{\Lambda, N}} p_{\Lambda, N}(\boldsymbol{n}) \sum_{k \in \Lambda^{*}} E_{n_{k}}\left(N_{a b}\right) .
$$

Proof. Let $p_{\Lambda, N}(\boldsymbol{k})=\sum_{\pi} p_{\Lambda, N}(\boldsymbol{k}, \pi)$. Since $\varrho_{a b}$ depends only on permutations, we have

$$
E_{\Lambda, N}\left(\varrho_{a b}\right)=\sum_{\boldsymbol{k} \in\left(\Lambda^{*}\right)^{N}} p_{\Lambda, N}(\boldsymbol{k}) \sum_{\pi \in \mathcal{S}_{N}} \varrho_{a b}(\pi) p_{\Lambda, N}(\pi \mid \boldsymbol{k}) .
$$

Actually, $\varrho_{a b}(\pi)$ depends only on the conjugacy class of $\pi$. In other words, we have, for any $\pi, \sigma \in \mathcal{S}_{N}$,

$$
\varrho_{a b}\left(\sigma^{-1} \pi \sigma\right)=\varrho_{a b}(\pi)
$$

It follows that

$$
\sum_{\pi \in \mathcal{S}_{N}} \varrho_{a b}(\pi) p_{\Lambda, N}(\pi \mid \boldsymbol{k})=\sum_{\pi \in \mathcal{S}_{N}} \varrho_{a b}(\pi) p_{\Lambda, N}(\pi \mid \sigma(\boldsymbol{k})) .
$$

Summing first over occupation numbers and then over compatible $\boldsymbol{k}$ 's, we get

$$
E_{\Lambda, N}\left(\varrho_{a b}\right)=\sum_{\boldsymbol{n} \in \mathcal{N}_{\Lambda, N}} p_{\Lambda, N}(\boldsymbol{n}) \sum_{\pi \in \mathcal{S}_{N}} \varrho_{a b}(\pi) p_{\Lambda, N}(\pi \mid \boldsymbol{k}) .
$$

Here, $\boldsymbol{k}$ is any vector that is compatible with $\boldsymbol{n}$.

A permutation $\pi$ such that $\pi(\boldsymbol{k})=\boldsymbol{k}$ (that is, $k_{\pi(i)}=k_{i}$ for all $i$ ) can be decomposed into permutations $\left(\pi_{k}\right)_{k \in \Lambda^{*}}$, where $\pi_{k}$ is a permutation of the $n_{k}$ indices $i$ such that $k_{i}=k$. Notice that

$$
N_{a b}(\pi)=\sum_{k \in \Lambda^{*}} N_{a b}\left(\pi_{k}\right), \quad r_{\ell}(\pi)=\sum_{k \in \Lambda^{*}} r_{\ell}\left(\pi_{k}\right) .
$$

Then

$$
p_{\Lambda, N}(\boldsymbol{k}, \pi)=\frac{1}{Y(\Lambda, N) N !} \prod_{k \in \Lambda^{*}} \mathrm{e}^{-n_{k} \varepsilon(k)} \mathrm{e}^{-\sum_{\ell} \alpha_{\ell} r_{\ell}(\pi)}
$$

if $\pi(\boldsymbol{k})=\boldsymbol{k} ;$ it is 0 otherwise. Also,

$$
p_{\Lambda, N}(\boldsymbol{k})=\frac{1}{Y(\Lambda, N) N !} \prod_{k \in \Lambda^{*}} \mathrm{e}^{-n_{k} \varepsilon(k)} h_{n_{k}} n_{k} !
$$


Then

$$
p_{\Lambda, N}(\pi \mid \boldsymbol{k})=\frac{p_{\Lambda, N}(\boldsymbol{k}, \pi)}{p_{\Lambda, N}(\boldsymbol{k})}=\prod_{k \in \Lambda^{*}} \frac{\mathrm{e}^{-\sum_{\ell} \alpha_{\ell} r_{\ell}(\pi)}}{h_{n_{k}} n_{k} !}
$$

if $\pi(\boldsymbol{k})=\boldsymbol{k}$, and 0 otherwise. Using (5.10),

$$
\begin{aligned}
E_{\Lambda, N}\left(\boldsymbol{\varrho}_{a b}\right) & =\frac{1}{V} \sum_{\boldsymbol{n} \in \mathcal{N}_{\Lambda, N}} p_{\Lambda, N}(\boldsymbol{n}) \sum_{\left(\pi_{k} \in \mathcal{S}_{n_{k}}\right)} \sum_{k \in \Lambda^{*}} \boldsymbol{N}_{a b}\left(\pi_{k}\right) \prod_{k^{\prime} \in \Lambda^{*}} \frac{\mathrm{e}^{-\sum_{\ell} \alpha_{\ell} r_{\ell}\left(\pi_{k^{\prime}}\right)}}{h_{n_{k^{\prime}}} n_{k^{\prime}} !} \\
& =\frac{1}{V} \sum_{\boldsymbol{n} \in \mathcal{N}_{\Lambda, N}} p_{\Lambda, N}(\boldsymbol{n}) \sum_{k \in \Lambda^{*}} E_{n_{k}}\left(N_{a b}\right) .
\end{aligned}
$$

In the light of Proposition 5.2, we can now focus on the quantity $p_{\Lambda, N}(\boldsymbol{n})$. Namely, our results from Section 2 imply that macroscopic cycles appear if and only if at least one mode is macroscopically occupied, i.e. iff $p_{\Lambda, N}\left(n_{k} \geqslant s N\right)>0$ uniformly in $N \in \mathbb{N}$ and $\Lambda$ such that $N=\rho \Lambda$.

We prove now that macroscopic occupation can occur only for $k=0$ and that it occurs if and only if $\rho$ is above the critical density $\rho_{\mathrm{c}}$ defined in (3.8). The first step is a result that gives detailed information about the limiting distribution of the random variable $n_{0} / V$.

Theorem 5.3. Let $\rho_{0}=\max \left(0, \rho-\rho_{\mathrm{c}}\right)$, with $\rho_{\mathrm{c}}$ the critical density defined in (3.8). Then for all $\lambda \geqslant 0$, we have

$$
\lim _{V \rightarrow \infty} E_{\Lambda, \rho V}\left(\mathrm{e}^{\lambda n_{0} / V}\right)=\mathrm{e}^{\lambda \rho_{0}}
$$

Proof. Our proof is based on the work of Buffet and Pulé [4] for the ideal Bose gas, see also [2]. We need to modify it due to the presence of cycle weights. We define

$$
Y(\Lambda, N, j)=\sum_{\boldsymbol{n} \in \mathcal{N}_{\Lambda, N}}\left(\prod_{k \in \Lambda^{*}} \mathrm{e}^{-\varepsilon(k) n_{k}} h_{n_{k}}\right) \times \begin{cases}h_{n_{0}+j} / h_{n_{0}} & \text { if } j \geqslant 0 \\ h_{\infty} / h_{n_{0}} & \text { if } j<0 .\end{cases}
$$

Then $Y(\Lambda, N, 0)=Y(\Lambda, N)$. Recall that $h_{\infty}=\lim _{n \rightarrow \infty} h_{n}$ is given in Proposition 2.3 (d) and that $0<h_{\infty}<\infty$. Then by (5.5),

$$
\begin{aligned}
p_{\Lambda, N}\left(n_{0} \geqslant j\right) & =\frac{1}{Y(\Lambda, N)} \sum_{\substack{n \in \mathcal{N}_{\Lambda, N} \\
n_{0} \geqslant j}} \prod_{k \in \Lambda^{*}} \mathrm{e}^{-n_{k} \varepsilon(k)} h_{n_{k}} \\
& =\frac{1}{Y(\Lambda, N)} \sum_{\substack{\boldsymbol{n} \in \mathcal{N}_{\Lambda, N-j} \\
h_{n_{0}}}} \frac{h_{n_{0}+j}}{\prod_{k \in \Lambda^{*}}} \mathrm{e}^{-n_{k} \varepsilon(k)} h_{n_{k}} \\
& =\frac{Y(\Lambda, N-j, j)}{Y(\Lambda, N)}
\end{aligned}
$$

for all $j \geqslant 0$. Using

$$
p_{\Lambda, N}\left(n_{0}=j\right)=p_{\Lambda, N}\left(n_{0} \geqslant j\right)-p_{\Lambda, N}\left(n_{0} \geqslant j+1\right),
$$

the change of summation index $j \mapsto N-j$ gives

$$
E_{\Lambda, N}\left(\mathrm{e}^{\nu n_{0}}\right)=\frac{\mathrm{e}^{\nu N}}{Y(\Lambda, N)} \sum_{j=0}^{N} \mathrm{e}^{-\nu j}(Y(\Lambda, j, N-j)-Y(\Lambda, j-1, N-j+1)) .
$$


Here we used the convention $Y(\Lambda,-1, N+1)=0$ and the fact that $p_{\Lambda, N}\left(n_{k} \geqslant N+1\right)=0$. We now fix $\rho \geqslant 0$ and put $\nu=\lambda / V$. As a first step we show that

$$
\lim _{V \rightarrow \infty} E_{\Lambda, \rho V}\left(\mathrm{e}^{\lambda n_{0} / V}\right)=1 \quad \text { for all } \rho \leqslant \rho_{\mathrm{c}} .
$$

Above, we wrote $\rho V$ instead of $\lfloor\rho V\rfloor$, and we will continue to do so in order to simplify the notation. To prove (5.19), we get from (5.18) that

$$
\begin{aligned}
& E_{\Lambda, \rho V}\left(\mathrm{e}^{\frac{\lambda n_{0}}{V}}\right)-\mathrm{e}^{-\frac{\lambda}{V}}= \\
& =\frac{\mathrm{e}^{\lambda \rho}}{Y(\Lambda, \rho V)} \sum_{j=0}^{\rho V} \mathrm{e}^{-\frac{\lambda j}{V}}(Y(\Lambda, j, \rho V-j)-Y(\Lambda, j-1, \rho V-j+1))-\mathrm{e}^{-\frac{\lambda}{V}} \\
& =\frac{\mathrm{e}^{\lambda \rho}}{Y(\Lambda, \rho V)}\left(\sum_{j=0}^{\rho V} \mathrm{e}^{-\frac{\lambda j}{V}} Y(\Lambda, j, \rho V-j)-\sum_{j=0}^{\rho V} \mathrm{e}^{-\frac{\lambda(j+1)}{V}} Y(\Lambda, j, \rho V-j)\right) \\
& =\frac{\mathrm{e}^{\lambda \rho}}{Y(\Lambda, \rho V)}\left(1-\mathrm{e}^{-\frac{\lambda}{V}}\right) \sum_{j=0}^{\rho V} \mathrm{e}^{-\frac{\lambda j}{V}} Y(\Lambda, j, \rho V-j)
\end{aligned}
$$

We need to show that $(5.20)$ converges to zero as $V \rightarrow \infty$. Recall the constant $B$ defined in Corollary 2.5. Since $Y(\Lambda, N, j) \leqslant B Y(\Lambda, N)$, we have

$$
\begin{aligned}
& E_{\Lambda, \rho V}\left(\mathrm{e}^{\frac{\lambda n_{0}}{V}}\right)-\mathrm{e}^{-\frac{\lambda}{V}} \leqslant B \frac{\mathrm{e}^{\lambda \rho}}{Y(\Lambda, \rho V)} \frac{\lambda}{V} \sum_{j=0}^{\rho V} \mathrm{e}^{-\frac{\lambda j}{V}} Y(\Lambda, j) \\
& =B \mathrm{e}^{\lambda \rho} \frac{\lambda}{V}\left(\sum_{j=0}^{(1-\varepsilon) \rho V} \mathrm{e}^{-\lambda j / V} \mathrm{e}^{-V\left(q_{\Lambda}(j / V)-q_{\Lambda}(\rho)\right)}+\sum_{j=0}^{\varepsilon \rho V} \mathrm{e}^{-\lambda(\rho V-j) / V} \frac{Y(\Lambda, \rho V-j)}{Y(\Lambda, \rho V)}\right)
\end{aligned}
$$

for any $\varepsilon>0$. Above, recall that $q_{\Lambda}(j / V)$ is the finite volume free energy given by (3.4). It follows from Theorem 3.4 that $q_{\Lambda}$ converges uniformly on compact intervals to the convex function $q$. By Theorem 3.3, $\rho \mapsto q(\rho)$ is strictly decreasing for $\rho<\rho_{\text {c }}$. For each $\varepsilon>0$ there is $b_{\varepsilon}>0$ such that $q_{\Lambda}(j / V)-q_{\Lambda}(\rho)>b_{\varepsilon}$ for all $V$ large enough, and all $j \leqslant(1-\varepsilon) \rho V$. So the first term in the bracket above is bounded by $(1-\varepsilon) \rho V \mathrm{e}^{-b_{\varepsilon} V}$ and thus converges to zero as $V \rightarrow \infty$. For the second term, we claim that $Y(\Lambda, \rho V-j) / Y(\Lambda, \rho V) \leqslant B$ for all $j$. This is proved by putting the extra $j$ particles into the zero mode $k=0$, or more formally through

$$
Y(\Lambda, N) \geqslant \sum_{\substack{n \in \mathcal{N}_{\Lambda, N} \\ n_{0} \geqslant j}} \prod_{k \in \Lambda^{*}} \mathrm{e}^{-\varepsilon(k) n_{k}} h_{n_{k}} \geqslant \frac{1}{B} Y(\Lambda, N-j) .
$$

Thus the second term, along with the prefactor $\mathrm{e}^{\lambda \rho} \lambda / V$, is bounded by $B^{2} \lambda \mathrm{e}^{\lambda \rho} \rho \varepsilon$. As $\varepsilon$ is arbitrarily small, we obtain (5.19).

We now turn to the case $\rho>\rho_{\mathrm{c}}$. We define the atomic measure

$$
\mu_{\Lambda, \rho}=C_{\Lambda} \sum_{j=0}^{\infty}(Y(\Lambda, j, \rho V-j)-Y(\Lambda, j-1, \rho V-j+1)) \delta_{j / V}
$$

on $\mathbb{R}^{+}$, with

$$
C_{\Lambda}=\left(\sum_{n \in \tilde{\mathcal{N}}_{\Lambda}} \prod_{k \neq 0} \mathrm{e}^{-\varepsilon(k) n_{k}} h_{n_{k}}\right)^{-1}
$$


here, we set

$$
\check{\mathcal{N}}_{\Lambda}=\left\{\boldsymbol{n} \in \mathcal{N}_{\Lambda}: n_{0}=0\right\}
$$

Later, we will also use the notation $\check{\mathcal{N}}_{\Lambda, N}=\left\{\boldsymbol{n} \in \mathcal{N}_{\Lambda, N}: n_{0}=0\right\}$. As we will see below, $C_{\Lambda}$ is a correct normalisation so that $\mu_{\Lambda, \rho}$ converges in the limit $V \rightarrow \infty$; it does not depend on $\rho$.

We rewrite (5.18) using $\mu_{\Lambda, \rho}$, which gives

$$
E_{\Lambda, \rho V}\left(\mathrm{e}^{\lambda n_{0} / V}\right)=\mathrm{e}^{\lambda \rho} \frac{\int 1_{[0, \rho]}(x) \mathrm{e}^{-\lambda x} \mu_{\Lambda, \rho}(\mathrm{d} x)}{\int 1_{[0, \rho]}(x) \mu_{\Lambda, \rho}(\mathrm{d} x)} .
$$

The strategy is to study the Laplace transform of $\mu_{\Lambda, \rho}$. It will be possible to take the limit $V \rightarrow \infty$. Putting the limiting measure in the right side of $(5.26)$, we will get the infinite volume limit of the left side. An advantage of this strategy is that several convergence issues are handled using standard theorems of analysis. The Laplace transform of $\mu_{\Lambda, \rho}$ is given by

$$
\begin{aligned}
\int_{0}^{\infty} \mathrm{e}^{-\lambda x} \mu_{\Lambda, \rho}(\mathrm{d} x) & =C_{\Lambda}\left(1-\mathrm{e}^{-\lambda / V}\right) \sum_{j=0}^{\infty} \mathrm{e}^{-\lambda j / V} Y(\Lambda, j, \rho V-j) \\
& =C_{\Lambda}\left(1-\mathrm{e}^{-\lambda / V}\right) \sum_{\boldsymbol{n} \in \mathcal{N}_{\Lambda}}\left(\prod_{k \in \Lambda^{*}} \mathrm{e}^{-(\varepsilon(k)+\lambda / V) n_{k}} h_{n_{k}}\right) \frac{\tilde{h}(\boldsymbol{n}, \rho V)}{h_{n_{0}}},
\end{aligned}
$$

with

$$
\tilde{h}(\boldsymbol{n}, N)= \begin{cases}h_{\left(N-\sum_{k \neq 0} n_{k}\right)} & \text { if } \sum_{k \in \Lambda^{*}} n_{k} \leqslant N, \\ h_{\infty} & \text { if } \sum_{k \in \Lambda^{*}} n_{k}>N .\end{cases}
$$

At this point, the idea in [4] and [2] was to factor out the contribution of the zero Fourier mode. This is not possible here because the factor $\tilde{h}(\boldsymbol{n}, \rho V)$ couples the modes. This difficulty can be circumvented by introducing

$$
\nu_{\Lambda}=C_{\Lambda} \sum_{j=0}^{\infty}(Y(\Lambda, j,-1)-Y(\Lambda, j-1,-1)) \delta_{j / N}
$$

and by writing

$$
\int_{0}^{\infty} \mathrm{e}^{-\lambda x} \mu_{\Lambda, \rho}(\mathrm{d} x)=\int_{0}^{\infty} \mathrm{e}^{-\lambda x} \nu_{\Lambda}(\mathrm{d} x)+\int_{0}^{\infty} \mathrm{e}^{-\lambda x}\left(\mu_{\Lambda, \rho}(\mathrm{d} x)-\nu_{\Lambda}(\mathrm{d} x)\right) .
$$

We now prove that the second term vanishes as $V \rightarrow \infty$. We have

$$
\begin{aligned}
& \int_{0}^{\infty} \mathrm{e}^{-\lambda x}\left(\mu_{\Lambda, \rho}(\mathrm{d} x)-\nu_{\Lambda}(\mathrm{d} x)\right)=C_{\Lambda}\left(1-\mathrm{e}^{-\frac{\lambda}{V}}\right) \sum_{j=0}^{\infty} \mathrm{e}^{-\frac{\lambda j}{V}}(Y(\Lambda, j, \rho V-j)-Y(\Lambda, j,-1)) \\
& =C_{\Lambda}\left(1-\mathrm{e}^{-\frac{\lambda}{V}}\right) \sum_{\substack{\boldsymbol{n} \in \mathcal{N}_{\Lambda} \\
\sum_{k} n_{k} \leqslant \rho V}}\left(\prod_{k \in \Lambda^{*}} \mathrm{e}^{-\left(\varepsilon(k)+\frac{\lambda}{V}\right) n_{k}} h_{n_{k}}\right) \frac{\tilde{h}(\boldsymbol{n}, \rho V)-h_{\infty}}{h_{n_{0}}} \\
& =C_{\Lambda}\left(1-\mathrm{e}^{-\frac{\lambda}{V}}\right) \sum_{n_{0} \geqslant 0} \mathrm{e}^{-\frac{\lambda n_{0}}{V}} \sum_{\substack{\boldsymbol{n} \in \mathcal{N}_{\Lambda} \\
\sum_{k \neq 0} n_{k} \leqslant \rho V-n_{0}}}\left(\prod_{k \neq 0} \mathrm{e}^{-\left(\varepsilon(k)+\frac{\lambda}{V}\right) n_{k}} h_{n_{k}}\right)\left(\tilde{h}(\boldsymbol{n}, \rho V)-h_{\infty}\right) .
\end{aligned}
$$


Now we maximize the second sum in the last line above over $n_{0}$, which obviously means putting $n_{0}=0$. The first sum is a geometric series and cancels the prefactor $\left(1-\mathrm{e}^{-\lambda / V}\right)$. As a result, (5.31) is less than

$$
C_{\Lambda} \sum_{\substack{\boldsymbol{n} \in \tilde{\mathcal{N}}_{\Lambda} \\ \sum_{k \neq 0} n_{k} \leqslant \rho V}}\left(\prod_{k \neq 0} \mathrm{e}^{-(\varepsilon(k)+\lambda / V) n_{k}} h_{n_{k}}\right)\left|\tilde{h}(\boldsymbol{n}, \rho V)-h_{\infty}\right| .
$$

The key observation now is that, by Proposition 2.4 (a), for given $\varepsilon>0$ there exists $m>0$ such that $\left|h_{m_{0}}-h_{\infty}\right|<\varepsilon$ whenever $m_{0}>m$. Then

$$
\sum_{\substack{\boldsymbol{n} \in \tilde{\mathcal{N}}_{\Lambda} \\ \sum_{k \neq 0} n_{k} \leqslant \rho V-m}}\left(\prod_{k \neq 0} \mathrm{e}^{-(\varepsilon(k)+\lambda / V) n_{k}} h_{n_{k}}\right)\left|\tilde{h}(\boldsymbol{n}, \rho V)-h_{\infty}\right| \leqslant \varepsilon \sum_{\boldsymbol{n} \in \tilde{\mathcal{N}}_{\Lambda}}\left(\prod_{k \neq 0} \mathrm{e}^{-\varepsilon(k) n_{k}} h_{n_{k}}\right) .
$$

Using the definition of $C_{\Lambda}$, we find that

$$
\begin{aligned}
\int_{0}^{\infty} \mathrm{e}^{-\lambda x}\left|\mu_{\Lambda, \rho}(\mathrm{d} x)-\nu_{\Lambda}(\mathrm{d} x)\right| & \leqslant \varepsilon+C_{\Lambda} \sum_{N=\rho V-m}^{\rho V}\left(h_{\rho V-N}-h_{\infty}\right) \check{Y}_{\lambda}(\Lambda, N) \\
& \leqslant \varepsilon+B C_{\Lambda} \sum_{N=\rho V-m}^{\rho V} \check{Y}_{0}(\Lambda, N)
\end{aligned}
$$

with

$$
\check{Y}_{\lambda}(\Lambda, N)=\sum_{\boldsymbol{n} \in \check{\mathcal{N}}_{\Lambda, N}}\left(\prod_{k \neq 0} \mathrm{e}^{-(\varepsilon(k)+\lambda / V) n_{k}} h_{n_{k}}\right)
$$

Note that $C_{\Lambda}^{-1}=\sum_{N \geqslant 1} \check{Y}_{0}(\Lambda, N)$, which suggests that the last term in 5.33 is small as the summation from $\rho V-m$ to $\rho V$ contains less terms than that giving $C_{\Lambda}^{-1}$. To prove this, let $\tilde{k}$ be one of the elements of $\Lambda^{*}$ closest to 0 . By putting $j$ particles into the mode $\tilde{k}$, we find that for any $j, N$

$$
\check{Y}_{0}(\Lambda, N+j) \geqslant \sum_{\boldsymbol{n} \in \check{\mathcal{N}}_{\Lambda, N}} \mathrm{e}^{-j \varepsilon(\tilde{k})} \frac{h_{n_{\tilde{k}}+j}}{h_{n_{\tilde{k}}}} \prod_{k \neq 0} \mathrm{e}^{-\varepsilon(k) n_{k}} h_{n_{k}} \geqslant B^{-1} \mathrm{e}^{-j \varepsilon(\tilde{k})} \check{Y}_{0}(\Lambda, N) .
$$

Thus for any $N$,

$$
C_{\Lambda}^{-1} \geqslant B^{-1} \check{Y}_{0}(\Lambda, N) \sum_{j=0}^{\infty} \mathrm{e}^{-j \varepsilon(\tilde{k})}=B^{-1} \check{Y}_{0}(\Lambda, N) \frac{1}{1-\mathrm{e}^{-\varepsilon(\tilde{k})}} \geqslant \frac{\check{Y}_{0}(\Lambda, N)}{B \varepsilon(\tilde{k})} .
$$

Inserting into 5.33 , we find that

$$
\int_{0}^{\infty} \mathrm{e}^{-\lambda x}\left|\mu_{\Lambda, \rho}(\mathrm{d} x)-\nu_{\Lambda}(\mathrm{d} x)\right| \leqslant \varepsilon+m B^{2} \varepsilon(\tilde{k}) .
$$

As $V \rightarrow \infty$ the second term vanishes since $\varepsilon(k)$ is continuous at 0 . Since $\varepsilon$ is arbitrarily small, the left side vanishes indeed in the limit.

Back to (5.30). For the first term, we can now follow the proof of Theorem A.1 in [2]. As above, we isolate the contribution of the zero mode and cancel it with the factor 
$1-\mathrm{e}^{-\lambda / V}$. Thus

$$
\int_{0}^{\infty} \mathrm{e}^{-\lambda x} \nu_{\Lambda}(\mathrm{d} x)=C_{\Lambda} h_{\infty} \sum_{\boldsymbol{n} \in \tilde{\mathcal{N}}_{\Lambda}}\left(\prod_{k \neq 0} \mathrm{e}^{-(\varepsilon(k)+\lambda / V) n_{k}} h_{n_{k}}\right) .
$$

Now

$$
\sum_{n \in \tilde{\mathcal{N}}_{\Lambda}}\left(\prod_{k \neq 0} \mathrm{e}^{-(\varepsilon(k)+\lambda / V) n_{k}} h_{n_{k}}\right)=\exp \left(\sum_{k \neq 0} \log \sum_{n \geqslant 0} \mathrm{e}^{-\left(\varepsilon(k)+\frac{\lambda}{V}\right) n} h_{n}\right) .
$$

By Proposition 2.3 (e), the logarithm in the exponential is equal to

$$
\sum_{j \geqslant 1} \mathrm{e}^{-\left(\varepsilon(k)+\frac{\lambda}{V}\right) j} \frac{\mathrm{e}^{-\alpha_{j}}}{j}=\sum_{j \geqslant 1} \mathrm{e}^{-\varepsilon(k) j} \frac{\mathrm{e}^{-\alpha_{j}}}{j}-\frac{1}{V} \int_{0}^{\lambda} \sum_{j \geqslant 1} \mathrm{e}^{-\left(\varepsilon(k)+\frac{s}{V}\right) j} \mathrm{e}^{-\alpha_{j}} \mathrm{~d} s .
$$

Using Proposition 2.3 (e) again, the first term in the inner bracket above is equal to $\sum_{k \neq 0} \log \sum_{n=0}^{\infty} \mathrm{e}^{-\varepsilon(k) n} h_{n}$, and thus it cancels $C_{\Lambda}$, while the second converges to $-\rho_{\mathrm{c}}$ as a Riemann sum for every $s \in[0, \lambda]$, when $V \rightarrow \infty$. Thus by dominated convergence in $s$, we obtain

$$
\lim _{V \rightarrow \infty} \int_{0}^{\infty} \mathrm{e}^{-\lambda x} \mu_{\Lambda, \rho}(\mathrm{d} x)=\lim _{V \rightarrow \infty} \int_{0}^{\infty} \mathrm{e}^{-\lambda x} \nu_{\Lambda}(\mathrm{d} x)=h_{\infty} \mathrm{e}^{-\rho_{\mathrm{c}}} .
$$

Thus by the general theory of Laplace transformations, $\mu_{\Lambda, \rho}$ converges to a delta peak of strength $h_{\infty}$ at $\rho_{\mathrm{c}}$. The claim of the theorem then follows from 5.26) for $\rho>\rho_{\mathrm{c}}$, and this completes the proof.

We prove now that the distribution of the random variable $\boldsymbol{n}$ shows typical behaviour. To that end we introduce the three sets

$$
\begin{aligned}
& A_{\epsilon}=\left\{\boldsymbol{n} \in \mathcal{N}_{\Lambda, N}:\left|\frac{n_{0}}{V}-\rho_{0}\right|<\epsilon\right\} \\
& B_{\epsilon, \delta}=\left\{\boldsymbol{n} \in \mathcal{N}_{\Lambda, N}: \sum_{\substack{\left.0<|k|<\delta \\
n_{k}<\epsilon V\right\}}} n_{k}<\sum_{\substack{k \in \Lambda^{*},|k| \geqslant \delta \\
n_{k}>M}} n_{k}<\epsilon V\right\} .
\end{aligned}
$$

Proposition 5.4. Under the assumptions of Theorem 3.5, for any density $\rho$ we have the following.

(a) For any $\epsilon>0, \lim _{V \rightarrow \infty} p_{\Lambda, \rho V}\left(A_{\epsilon}\right)=1$.

(b) Suppose that $\rho_{\mathrm{c}}<\infty$. For any $\epsilon>0$, there exists $\delta_{\epsilon}$ such that $p_{\Lambda, \rho V}\left(B_{\epsilon, \delta_{\epsilon}}\right)>1-\epsilon$ for $V$ large enough.

(c) For any $\epsilon, \delta>0$, there exists $M_{\epsilon, \delta}$ such that $\lim _{V \rightarrow \infty} p_{\Lambda, \rho V}\left(C_{\epsilon, \delta, M_{\epsilon, \delta}}\right)=1$.

The restriction for finite $\rho_{\mathrm{c}}$ in item (b) should not be there - but we cannot prove the claim without it. This is the only reason why Theorem 3.5 does not hold when the critical density is infinite.

Proof. The claim (a) follows from Theorem 5.3.

$$
\lim _{V \rightarrow \infty} p_{\Lambda, \rho V}\left(A_{\epsilon}\right)=\lim _{V \rightarrow \infty} E_{\Lambda, \rho V}\left(1_{\left[\rho_{0}-\epsilon, \rho_{0}+\epsilon\right]}\left(\frac{n_{0}}{V}\right)\right)=\int 1_{\left[\rho_{0}-\epsilon, \rho_{0}+\epsilon\right]}(s) \delta_{\rho_{0}}(s) \mathrm{d} s=1 .
$$


We now get a bound on the probability of a given occupation number. Recall the constant $B$ of Corollary 2.5.

$$
\begin{aligned}
p_{\Lambda, N}\left(n_{k} \geqslant i\right) & =\frac{1}{Y(\Lambda, N)} \sum_{\boldsymbol{n} \in \mathcal{N}_{\Lambda, N-i}} \mathrm{e}^{-\varepsilon(k) i}\left(\prod_{k^{\prime} \in \Lambda^{*}} \mathrm{e}^{-\varepsilon\left(k^{\prime}\right) n_{k^{\prime}} h_{n_{k^{\prime}}}}\right) \frac{h_{n_{k}+i}}{h_{n_{k}}} \\
& \leqslant B \mathrm{e}^{-\varepsilon(k) i} \frac{Y(\Lambda, N-i)}{Y(\Lambda, N)}
\end{aligned}
$$

We also have that $\frac{Y(\Lambda, N-i)}{Y(\Lambda, N)} \leqslant B$, see 5.22 . Then

$$
E_{\Lambda, N}\left(n_{k}\right)=\sum_{i \geqslant 1} p_{\Lambda, N}\left(n_{k} \geqslant i\right) \leqslant \frac{B^{2}}{\mathrm{e}^{\varepsilon(k)}-1} .
$$

By Markov inequality,

$$
p_{\Lambda, N}\left(B_{\epsilon, \delta_{\epsilon}}^{\mathrm{c}}\right) \leqslant \frac{B^{2}}{\epsilon V} \sum_{0<|k|<\delta_{\epsilon}} \frac{1}{\mathrm{e}^{\varepsilon(k)}-1} \stackrel{V \rightarrow \infty}{\longrightarrow} \frac{B^{2}}{\epsilon} \int_{0<|k|<\delta_{\epsilon}} \frac{\mathrm{d} k}{\mathrm{e}^{\varepsilon(k)}-1} .
$$

The integral converges because the critical density $(3.8)$ is finite. It is possible to choose $V$ large enough and $\delta_{\epsilon}$ small enough so that $p_{\Lambda, N}\left(B_{\epsilon, \delta_{\epsilon}}^{\mathrm{c}}\right)<\epsilon$.

For the claim (c), we use

$$
p_{\Lambda, N}\left(C_{\epsilon, \delta, M}^{\mathrm{c}}\right) \leqslant \sum_{m \geqslant 1} \frac{1}{m !} \sum_{\substack{k_{1}, \ldots, k_{m} \in \Lambda^{*} \\\left|k_{i}\right|>\delta}} \frac{1}{Y(\Lambda, N)} \sum_{\substack{n \in \mathcal{N}_{\Lambda, N} \\ n_{k_{i}}>M \\ \sum_{i} n_{k_{i}} \geqslant \epsilon N}} \prod_{k \in \Lambda^{*}} \mathrm{e}^{-\varepsilon(k) n_{k}} h_{n_{k}}
$$

For given $k_{1}, \ldots, k_{m}$, we have

$$
\begin{aligned}
\sum_{\substack{\boldsymbol{n} \in \mathcal{N}_{\Lambda, N} \\
n_{k_{i}}>M \\
\sum n_{k_{i}}>\epsilon N}} \prod_{k \in \Lambda^{*}} \mathrm{e}^{-\varepsilon(k) n_{k}} h_{n_{k}}= \\
\quad=\sum_{\substack{n_{1}, \ldots, n_{m}>M \\
\epsilon N \leqslant \sum n_{i} \leqslant N}} \sum_{n^{\prime} \in \mathcal{N}_{\Lambda, N}-\sum n_{i}}\left(\prod_{k \in \Lambda^{*}} \mathrm{e}^{-\varepsilon(k) n_{k}^{\prime}} h_{n_{k}^{\prime}}\right)\left(\prod_{i=1}^{m} \mathrm{e}^{-\varepsilon\left(k_{i}\right) n_{i}} \frac{h_{n_{k_{i}}^{\prime}+n_{i}}}{h_{n_{k_{i}}^{\prime}}}\right) .
\end{aligned}
$$

We can bound the last ratio by $B$. Then

$$
p_{\Lambda, N}\left(C_{\epsilon, \delta, M}^{\mathrm{c}}\right) \leqslant \sum_{m \geqslant 1} \frac{B^{m}}{m !} \sum_{\substack{k_{1}, \ldots, k_{m} \in \Lambda^{*} \\\left|k_{i}\right|>\delta}} \sum_{\substack{n_{1}, \ldots, n_{m}>M \\ \epsilon N \leqslant \sum n_{i} \leqslant N}} \frac{Y\left(\Lambda, N-\sum n_{i}\right)}{Y(\Lambda, N)} \prod_{i=1}^{m} \mathrm{e}^{-\varepsilon\left(k_{i}\right) n_{i}}
$$

We bound the ratio of partition functions by $B$, see $(5.22)$, and we bound one half of $\varepsilon\left(k_{i}\right)$ by one half of

$$
\varepsilon_{0}=\min _{|k|>\delta} \varepsilon(k)>0
$$


Then, since $\sum n_{i} \geqslant \epsilon N$, we have

$$
\begin{aligned}
p_{\Lambda, N}\left(C_{\epsilon, \delta, M}^{\mathrm{c}}\right) & \leqslant \mathrm{e}^{-\frac{1}{2} \varepsilon_{0} \epsilon N} \sum_{m \geqslant 1} \frac{B^{m+1}}{m !}\left(\sum_{|k|>\delta} \sum_{n>M} \mathrm{e}^{-\frac{1}{2} \varepsilon(k) n}\right)^{m} \\
& \leqslant B \exp \left\{-V\left[\frac{1}{2} \varepsilon_{0} \epsilon \rho-B \mathrm{e}^{-\frac{1}{2} \varepsilon_{0} M} \frac{1}{V} \sum_{|k|>\delta} \frac{1}{\mathrm{e}^{\frac{1}{2} \varepsilon(k)}-1}\right]\right\} .
\end{aligned}
$$

We recognise a Riemann sum which is bounded uniformly in $V$. If $M$ is large enough (depending on $\epsilon$ and $\varepsilon_{0}$, hence on $\delta$ ), the term in the bracket is positive and everything vanishes in the limit $V \rightarrow \infty$.

We are now equipped for the proof of Theorem 3.5 . We use Propositions 5.2 and 5.4 . and also Theorem 2.1.

Proof of Theorem 3.5. From Proposition 5.2 we can split

$$
E_{\Lambda, N}\left(\varrho_{a, b}\right)=\frac{1}{V} \sum_{\boldsymbol{n} \in \mathcal{N}_{\Lambda, N}} p_{\Lambda, N}(\boldsymbol{n})\left[E_{n_{0}}\left(N_{a, b}\right)+\sum_{0<|k|<\delta} E_{n_{k}}\left(N_{a, b}\right)+\sum_{|k| \geqslant \delta} E_{n_{k}}\left(N_{a, b}\right)\right] .
$$

We treat the cases separately. By Proposition 5.4 (a), we can restrict the sum to $\boldsymbol{n} \in A_{\epsilon}$ with arbitrarily small $\epsilon$. Then for any $\eta(V)$ such that $\eta(V) / V \rightarrow 0$,

$$
\lim _{V \rightarrow \infty} \sum_{\boldsymbol{n} \in \mathcal{N}_{\Lambda, \rho V}} p_{\Lambda, \rho V}(\boldsymbol{n}) \frac{1}{V} E_{n_{0}}\left(N_{1, \eta(V)}\right)=\lim _{V \rightarrow \infty} \frac{1}{V} E_{\rho_{0} V}\left(N_{1, \eta(V)}\right),
$$

which is zero by Theorem 2.1. On the other hand, for the same reasons we have

$$
\begin{aligned}
\lim _{V \rightarrow \infty} \sum_{\boldsymbol{n} \in \mathcal{N}_{\Lambda, \rho V}} p_{\Lambda, \rho V}(\boldsymbol{n}) \frac{1}{V} E_{n_{0}}\left(N_{\eta(V), s V}\right) & =\lim _{V \rightarrow \infty} \frac{1}{V} E_{\rho_{0} V}\left(N_{\eta(V), s V}\right) \\
& = \begin{cases}s & \text { if } 0 \leqslant s \leqslant \rho_{0}, \\
\rho_{0} & \text { if } s \geqslant \rho_{0} .\end{cases}
\end{aligned}
$$

Next we use Proposition 5.4 (b) to show that the modes $0<|k|<\delta$ contribute a vanishing amount. Indeed, we can find arbitrarily small $\epsilon$ and $\delta=\delta_{\epsilon}$ such that

$$
\frac{1}{V} \sum_{\boldsymbol{n} \in \mathcal{N}_{\Lambda, N}} p_{\Lambda, N}(\boldsymbol{n}) \sum_{0<|k|<\delta} E_{n_{k}}\left(N_{1, \rho V}\right) \leqslant \epsilon+\frac{1}{V} \sum_{\boldsymbol{n} \in B_{\epsilon, \delta}} p_{\Lambda, N}(\boldsymbol{n}) \sum_{0<|k|<\delta} E_{n_{k}}\left(N_{1, \rho V}\right) \leqslant 2 \epsilon .
$$

Therefore we can neglect those modes in 5.52 without changing the result in the limit $V \rightarrow \infty$.

There remain the modes $|k|>\delta$. By Proposition 5.4 (c) we can restrict the sum over $\boldsymbol{n} \in C_{\epsilon, \delta, M_{\epsilon, \delta}}$. And because of the definition of $C_{\epsilon, \delta, M_{\epsilon, \delta}}$, we get

$$
\lim _{V \rightarrow \infty} \frac{1}{V} \sum_{\boldsymbol{n} \in C_{\epsilon, \delta, M_{\epsilon, \delta}}} p_{\Lambda, \rho V}(\boldsymbol{n}) \sum_{|k|>\delta} E_{n_{k}}\left(N_{\eta(V), \rho V}\right) \leqslant \epsilon .
$$

The estimates obtained above prove the second and the third claim of Theorem 3.5 - and therefore also the first claim, since the fraction of points in microscopic cycles is obviously equal to the total density, minus the fraction of points in mesoscopic and macroscopic cycles. 


\section{Appendix A. Thermodynamic POTEntials With PERIODIC BOUNDARY CONDITIONS}

We clearly have $Y^{\text {per }}(\Lambda, N) \geqslant Y(\Lambda, N)$, so that

$$
q_{\Lambda}^{\mathrm{per}}(\rho) \leqslant q_{\Lambda}(\rho)
$$

It is thus enough to show that $\lim \inf q_{\Lambda_{n}}^{\text {per }}$ converges to $q$. First we establish some continuity property for $q_{\Lambda}^{\text {per }}$.

Lemma A.1. For any $\Lambda$ and any $\eta>0$, we have

$$
q_{\Lambda}^{\mathrm{per}}(\rho+\eta) \leqslant q_{\Lambda}^{\mathrm{per}}(\rho)+\frac{\log B}{|\Lambda|}+\eta \varepsilon_{\Lambda}(0)
$$

with $B$ the constant of Corollary 2.5.

Proof. Let $N=\rho|\Lambda|$ and $M=\eta|\Lambda|$. The partition function with periodic boundary conditions is

$$
\begin{aligned}
Y^{\mathrm{per}}(\Lambda, N+M) & =\sum_{\boldsymbol{n} \in \mathcal{N}_{\Lambda, N+M}} \prod_{k \in \Lambda^{*}} \mathrm{e}^{-n_{k} \varepsilon_{\Lambda}(k)} h_{n_{k}} \\
& \geqslant \sum_{\boldsymbol{n} \in \mathcal{N}_{\Lambda, N}} \mathrm{e}^{-\left(n_{0}+M\right) \varepsilon_{\Lambda}(0)} h_{n_{0}+M} \prod_{k \in \Lambda^{*} \backslash\{0\}} \mathrm{e}^{-n_{k} \varepsilon_{\Lambda}(k)} h_{n_{k}} .
\end{aligned}
$$

To get the second line we restricted the sum over occupation numbers to those with $n_{0} \geqslant M$. We know from Corollary 2.5 that $h_{n_{0}+M} \geqslant h_{n_{0}} / B$ and we get the lemma.

Proof of Theorem 3.4. We can derive an expression for the pressure $p_{\Lambda}^{\text {per }}$ like in the proof of Theorem 3.1, namely

$$
p_{\Lambda}^{\text {per }}(\mu)=\frac{1}{|\Lambda|} \sum_{n \geqslant 1} \frac{\mathrm{e}^{\mu n-\alpha_{n}}}{n} \sum_{k \in \Lambda^{*}} \mathrm{e}^{-n \varepsilon_{\Lambda}(k)} .
$$

The last sum is less than $\sum_{k} \mathrm{e}^{-\varepsilon_{\Lambda}(k)}=\mathrm{e}^{-\xi(0)}$. Then $p_{\Lambda}^{\text {per }}$ converges to the expression for $p$ in Theorem 3.1 by dominated convergence.

We turn to the free energy with periodic boundary conditions. Let us define

$$
q^{\text {per }}(\rho)=\liminf _{n \rightarrow \infty} q_{\Lambda_{n}}^{\text {per }}(\rho) .
$$

Suppose that there exists $\rho^{*}$ such that $q^{\mathrm{per}}\left(\rho^{*}\right)<q\left(\rho^{*}\right)$. It follows from Lemma A.1 that there exists an interval $I$ close to $\rho^{*}$ and an $\eta>0$ such that

$$
q_{\Lambda}^{\text {per }}(\rho)<q_{\Lambda}(\rho)-\eta
$$

for all $\rho \in I$. Using (A.1), we find that the pressure satisfies

$$
p_{\Lambda}^{\text {per }}(\mu) \geqslant \sum_{N \geqslant 0} \mathrm{e}^{\mu N} \mathrm{e}^{-q_{\Lambda}\left(\frac{N}{V}\right)+\eta 1_{I}\left(\frac{N}{V}\right)}
$$

Then the infinite volume limit of $p_{\Lambda}^{\mathrm{per}}(\mu)$ is larger than the Legendre transform of $q-\eta \chi_{I}$, hence larger than $p(\mu)$ for some $\mu<0$. This contradicts the first claim of Theorem 3.4 . This shows that $q_{\Lambda}^{\text {per }}$ converges pointwise to $q$. The uniform convergence on compact sets follows from A.1], the uniform convergence of $q_{\Lambda}$, and Lemma A.1.

Acknowledgments: We are grateful to the referees for useful comments. D.U. is grateful to the hospitality of the Erwin Schrödinger Insitut of Vienna, the University of Geneva, ETH Zürich, and the Center of Theoretical Studies of Prague, where parts of this project were carried forward. V.B. is supported 
by the EPSRC fellowship EP/D07181X/1 and D.U. is supported in part by the grant DMS-0601075 of the US National Science Foundation.

\title{
REFERENCES
}

[1] J. Baik, P. Deift, K. Johannson, On the distribution of the length of the longest increasing subsequence of random permutations J. Amer. Math. Soc. 12, 1119-1178 (1999)

[2] V. Betz, D. Ueltschi, Spatial random permutations and infinite cycles, Commun. Math. Phys. 285, 469-501 (2009)

[3] V. Betz, D. Ueltschi, Y. Velenik, Random permutations with cycle weights, in preparation

[4] E. Buffet, J. V. Pulé, Fluctuation properties of the imperfect Bose gas, J. Math. Phys. 24, 1608-1616 (1983)

[5] W. J. Ewens, The sampling theory of selectively neutral alleles, Theoret. Populations Biol. 3, 87-112 $(1972)$

[6] S. Feng, F. M. Hoppe, Large deviation principles for some random combinatorial structures in population genetics and Brownian motion, Ann. Appl. Probab. 8, 975-994 (1998)

[7] P. Ferrari, M. Prähofer, H. Spohn, Stochastic growth in one dimension and Gaussian multi-matrix models, XIVth International Congress on Mathematical Physics, World Scientific 2005

[8] R. P. Feynman, Atomic theory of the $\lambda$ transition in Helium, Phys. Rev. 91, 1291-1301 (1953)

[9] D. Gandolfo, J. Ruiz, D. Ueltschi, On a model of random cycles, J. Stat. Phys. 129, 663-676 (2007)

[10] A. Okounkov, The uses of random partitions, XIVth International Congress on Mathematical Physics, 379-403, World Scientific 2005

[11] J. Pitman, Exchangeable and partially exchangeable random partitions, Probab. Theory Rel. Fields 102, 145-158 (1995)

[12] D. Ruelle, Statistical Mechanics: Rigorous Results, World Scientific (1999)

[13] L. A. Shepp and S. P. Lloyd, Ordered Cycle Lengths in a Random Permutation, Transactions of the American Mathematical Society, Vol. 121, No. 2, 340-357 (1966)

[14] O. Schramm, Compositions of random transpositions, http://arxiv.org/abs/math/0404356v3 (2004)

[15] A. Sütő, Percolation transition in the Bose gas, J. Phys. A 26, 4689-4710 (1993)

[16] A. Sütö, Percolation transition in the Bose gas II, J. Phys. A 35, 6995-7002 (2002)

\author{
Volker Betz and Daniel Ueltschi \\ Department of Mathematics \\ UNIVERSITY OF WARWICK \\ COVEntry, CV4 7AL, ENGLAND \\ http://www.maths.warwick.ac.uk/ betz/ \\ http://www.ueltschi.org \\ E-mail address: v.m.betz@warwick.ac.uk, daniel@ueltschi.org
}

